\title{
19. PHYSICAL PROPERTIES OF SITE 762: A COMPARISON OF SHIPBOARD AND SHORE-BASED LABORATORY RESULTS ${ }^{1}$
}

\author{
David K. O'Brien ${ }^{2,3}$ and Murli H. Manghnani ${ }^{2}$
}

\begin{abstract}
Compressional velocity $\left(V_{p}\right)$, shear velocity $\left(V_{s}\right)$, compressional quality factor $\left(Q_{p}\right)$, electrical resistivity $\left(\rho^{\prime}\right)$, bulk density $\left(\rho_{b}\right)$, grain density $\left(\rho_{g}\right)$, and porosity $(\phi$ were measured in our shore-based laboratory for 49 consolidated sediment samples from Hole $762 \mathrm{C}$. The results are compared with shipboard data.

Shore-based $V_{p}$ values agree well with shipboard $V_{p}$ data except in the range 670-820 meters below seafloor, where a shipboard calibration problem occurred. Shipboard sonic log data are an average of $0.3 \mathrm{~km} / \mathrm{s}$ higher than shore-based $V_{p}$ values because of in-situ overburden pressure.

Shore-based $\rho_{g}$ and $\phi$ values are generally in agreement with shipboard data. However, shipboard $\rho_{b}$ values are consistently higher than shore-based data. This discrepancy is because the helium-displacement pycnometer used aboard ship gives erroneously low volumes for wet samples, which are then used in bulk density calculations. Correct shipboard wet sample volumes can be calculated by adding the difference between the wet and dry sample weights to the dry sample volume. The corrected shipboard $\rho_{b}$ values are in agreement with shore-based data. We recommend that the Ocean Drilling Program use this calculation in place of the pycnometer wet volumes.

The chalks show a negative velocity gradient between 600 and $720 \mathrm{mbsf}$, though there is no apparent change in lithology. In absence of overpressuring and mineralogical changes, the negative gradient is probably caused by increasing porosity due to the change in microstructure of the sediment over this depth interval.
\end{abstract}

\section{INTRODUCTION}

The variations in physical, acoustic, and diagenetic properties of deep-sea sediments as a function of depth are important in the development of geoacoustic models (Hamilton, 1980; Milholland et al., 1980). Here, we report physical properties measured in our Hawaii sediment acoustics laboratory for 49 consolidated samples from Ocean Drilling Program (ODP) Hole 762C. These properties are shear velocity $\left(V_{s}\right)$, compressional attenuation (in terms of its inverse, the quality factor $\left.Q_{p}\right)$, and electrical resistivity $\left(\rho^{\prime}\right)$. These measurements are not generally performed aboard the JOIDES Resolution. In addition, compressional velocity $\left(V_{p}\right)$, bulk density $\left(\rho_{b}\right)$, grain density $\left(\rho_{g}\right)$, and porosity $(\phi)$ have been remeasured using alternative methods to those used aboard ship. Because different techniques were used for shipboard and shore-based laboratory measurements, it is important to compare these measurements on the same sediment samples or adjacent samples so that shore-based measurements can be confidently compared to various other shipboard measurements.

\section{SAMPLES}

Sediment samples used in this study are from Hole $762 \mathrm{C}$, located on the Exmouth Plateau, part of the northwestern Australia continental margin (Fig. 1). The plateau was connected to present-day India until breakup occurred in the early Cretaceous (Neocomian) due to seafloor spreading (Exon et al., 1982). The sediments of Site 762 consist of a post-breakup pelagic carbonate sequence overlying a pre-breakup claystone sequence. The post-breakup carbonate sediments consist of

\footnotetext{
1 von Rad, U., Haq, B. U., et al., 1992. Proc. ODP, Sci. Results, 122: College Station, TX (Ocean Drilling Program). U.S.A.

2 Hawaii Institute of Geophysics, 2525 Correa Road, Honolulu, HI 96822 ,

${ }^{3}$ Current address: America North Inc., 201 E. 56th Avenue, Anchorage, AK 99518, U.S.A.
}

nannofossil ooze (Unit I, 0-181.5 mbsf), nannofossil ooze and chalk transitional sediments (Unit II, 181.5-265.0 mbsf), and nannofossil chalk. The nannofossil chalks are divided into Unit III (265.0-554.8 mbsf), which has a low clay content, and Unit IV (554.8-838.5 mbsf), which has a higher clay content, as observed in smear slides. The pre-breakup claystones consist of a hemipelagic calcareous claystone sequence (Unit $\mathrm{V}, 838.5-848.5 \mathrm{mbsf}$ ) and a deltaic silty claystone sequence (Unit VI, 848.5-940.0 mbsf).

The samples in this study are consolidated and range from 371.69 mbsf (Sample 122-762C-23X-2, 68-70 cm) to 931.61 mbsf (Sample 122-762C-91X-2, 10-12 cm). Samples were located directly adjacent to or nearby shipboard physical properties samples. Each sample was wrapped in wet tissue paper, placed in a vial filled with seawater, and sealed in a plastic bag aboard ship to prevent moisture loss. Prior to shore-based measurements, each sample was cut into a cube (approximately $1.78 \mathrm{~cm}$ on an edge), with edges parallel and perpendicular to the core axis, using an Buehler Isomet low-speed saw with water as the lubricating fluid. Bedding was present in 22 of the samples and was approximately horizontal.

\section{EXPERIMENTAL PROCEDURES}

\section{Compressional and Shear Velocity}

Compressional velocity $\left(V_{p}\right)$ measurements were made in the horizontal $\left(V_{p h}\right)$ and vertical $\left(V_{p v}\right)$ directions with respect to the core axis and at ambient temperature and pressure. $V_{p}$ was measured using the pulse transmission technique in conjunction with a Tektronix 7894 digital oscilloscope and an HP microcomputer. The waveform traces on the oscilloscope for the input pulse and sample signal were first digitized. A computer program analyzed the waveforms, automatically placed cursors on the input pulse and sample waveform on the oscilloscope screen at the first break from horizontal, and determined the traveltime between the cursors. The system was calibrated using varying lengths of plexiglass rods by 


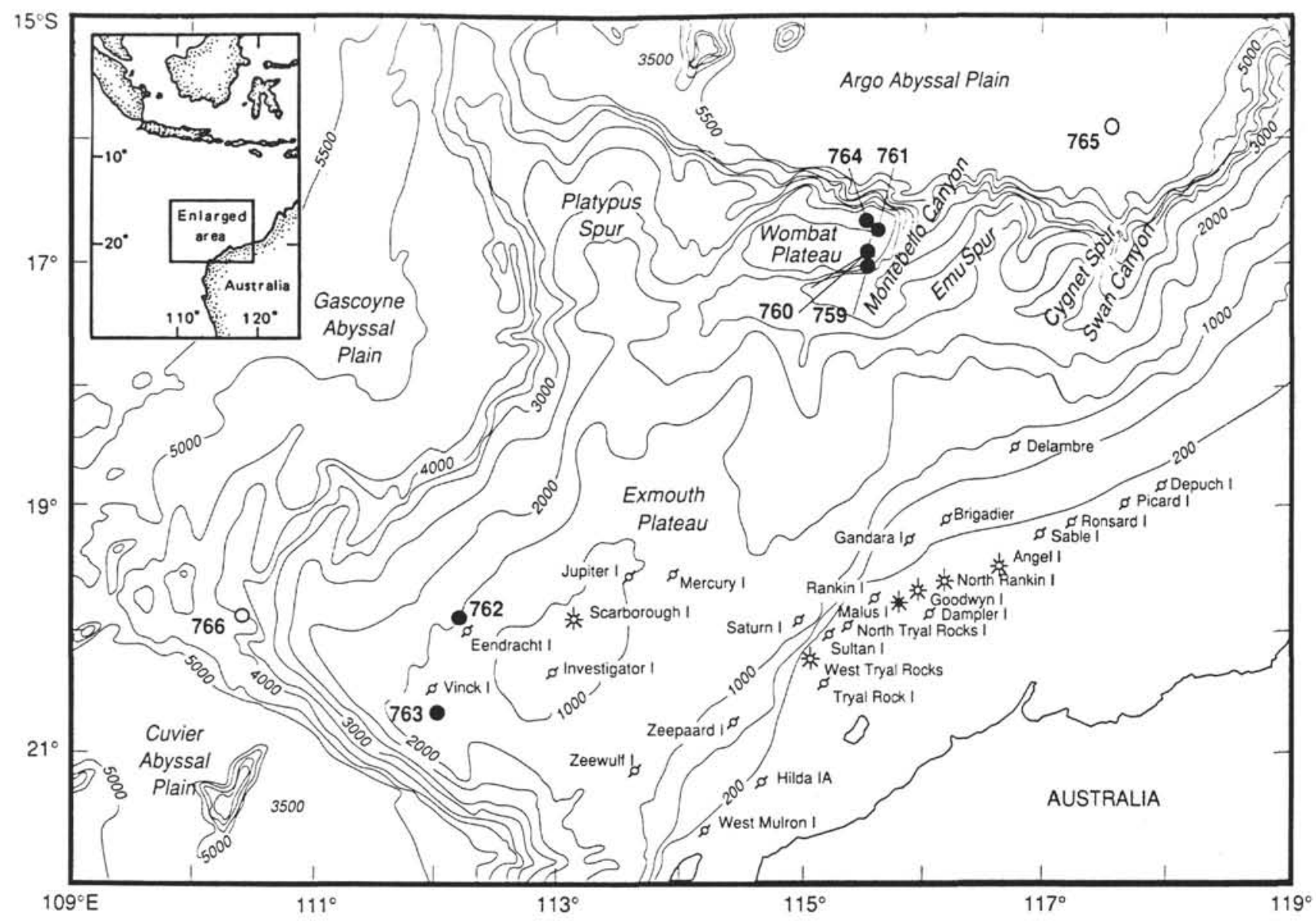

Figure 1. Bathymetric map of the Exmouth Plateau showing locations of ODP sites (closed circles are Leg 122 sites, open circles are Leg 123 sites) and commercial wells. Bathymetry is shown in meters. Modified from Haq, von Rad, O'Connell, et al. (1990).

making a plot of rod length vs. traveltime and linearly extrapolating to zero rod length to determine the system delay time, which was then subtracted from all sample traveltimes. We used $1-\mathrm{MHz}, 0.5$-in. diameter metaniobate $P$-wave transducers (Panametrics V103) with water as the acoustic coupling fluid.

Shear-wave $\left(V_{s}\right)$ velocities were measured similarly, but because there are always compressional waves in a shearwave arrival signal, the computer could not clearly distinguish the first arrival of the shear-wave signal. Therefore, the cursor was placed manually on the shear-wave first arrival. We used 1-MHz, 0.5 -in. diameter PZT $S$-wave transducers (Harisonic S0108) with water as a coupling fluid.

\section{Attenuation}

The compressional quality factor, $Q_{p}$, was measured in both horizontal $\left(Q_{p h}\right)$ and vertical $\left(Q_{p v}\right)$ directions by using the spectral ratio technique (Toksoz et al., 1979; Johnston and Toksoz, 1980). The amplitude of the $P$-wave signal from a consolidated sediment sample cube was compared with that of a reference material (e.g., aluminum) with high $Q(\approx 150,000$; Zamanek and Rudnick, 1961). The reference and sample cubes were of the same size.

Theoretical and experimental aspects of the spectral ratio technique are discussed elsewhere (Toksoz et al., 1979; Johnston and Toksoz, 1980). Briefly, the first 1.5 wavelengths of the $P$-wave signals from both the sample and the reference are digitized (successive wavelengths contain reverberations from within the samples). The fast-Fourier transform (FFT) of the reference signal is divided by the FFT of the sample, and the natural logarithm is then calculated to obtain the spectral ratio. It is assumed that attenuation is a linear function of frequency near the resonance frequency of the transducers (1 $\mathrm{MHz}$ (Toksoz et al., 1979). A line can be fit to the data near this frequency, and the value of $Q_{p}$ is determined from the slope of this line:

$$
\text { Slope }=\frac{\ln \left(A_{1} / A_{2}\right)}{f}=\frac{\pi L}{Q_{p} V_{p}},
$$

where $A_{1}$ is the amplitude of the reference signal, $A_{2}$ is the amplitude of the sample signal, $f$ is frequency, $L$ is sample length, and $V_{p}$ is the compressional velocity.

\section{Electrical Resistivity}

The four-electrode technique (Olhoeft, 1979, 1980) was used to measure electrical resistivity $\left(\rho^{\prime}\right)$. The technique and theoretical background have been discussed elsewhere (Collett and Katsube, 1973; Katsube, 1977; Olhoeft, 1979). Electrical resistivity was measured in horizontal and vertical directions at frequencies of $0.1,1.0,10,100,1000$, and 10000 $\mathrm{Hz}$.

\section{Density and Porosity}

Archimedes' principle of buoyancy (Ference et al., 1956; Johnson, 1979) was used to determine bulk $\left(\rho_{b}\right)$ and grain $\left(\rho_{g}\right)$ densities and porosity $(\phi)$. Each sample was weighed wet in 
air and while buoyant in a basket submerged in a cup of distilled water. The sample was then dried in an oven at $105^{\circ} \mathrm{C}$ for $24 \mathrm{hr}$ and then allowed to cool in a desiccator under vacuum for several hours before being weighed dry. The basic equations for these physical properties are

$$
\begin{gathered}
\text { Bulk density }=\frac{W_{w a} \rho_{w a t e r}}{W_{w a}-W_{w l}}, \\
\text { Grain density }=\frac{W_{d a} \rho_{w a t e r}}{W_{d a}-W_{w l}}, \text { and } \\
\text { Porosity }=\frac{W_{w a}-W_{d a}}{W_{w a}-W_{w l}},
\end{gathered}
$$

where $W_{d a}$ is dry weight in air, $W_{w a}$ is wet weight in air, $W_{w l}$ is wet weight in distilled water while buoyant, and $\rho_{\text {water }}$ is density of distilled water.

The above equations are derived using the following relations: volume of saturated sample $=\left(W_{w a}-W_{w l}\right) / \rho_{\text {water }}$; volume of water in sample $=\left(W_{w a}-W_{d a}\right) / \rho_{\text {water }}$; and volume of grains in sample $=$ volume of saturated sample - volume of water.

However, because salts are deposited in pores when a sample is dried, the equations for grain density and porosity must be modified to compensate for the extra salt in the dry volume and dry weight for each sample. The salt corrections are derived as follows:

Salinity of seawater is assumed to be 35 parts per thousand (ppt). Therefore, there is $0.035 \mathrm{~g}$ of salt per $1 \mathrm{~g}$ of seawater, or $0.965 \mathrm{~g}$ of freshwater per $1 \mathrm{~g}$ of seawater. The weight of halite precipitated out of seawater in a dry sample $\left(W_{h}\right)$ can be determined by

$$
\begin{aligned}
& W_{h}=\text { weight of seawater in sample - } \\
& \text { weight of freshwater in sample }
\end{aligned}
$$

$$
=\left(W_{w a}-W_{d a}\right)\left(\frac{1}{0.965}-1\right) .
$$

The density of halite is taken as $2.165 \mathrm{~g} / \mathrm{cm}^{3}$, seawater as 1.025 $\mathrm{g} / \mathrm{cm}^{3}$ (at $15^{\circ} \mathrm{C}$ ), and distilled water as 0.9986 (at $18^{\circ} \mathrm{C}$ ) (Weast and Astle, 1981). The modified equations for grain density and porosity are as follows:

$$
\begin{gathered}
\text { Grain density }=\frac{\left(W_{d a}-W_{h}\right) \rho_{\text {water }}}{\left(W_{d a}-W_{w l}\right)-\left(\left(W_{h} \rho_{\text {water }}\right) / 2.165\right)} ; \\
\text { Porosity }=\frac{\left(W_{w a}-W_{d a}\right) / 1.025}{\left(W_{w a}-W_{w l}\right) / \rho_{\text {water }}} .
\end{gathered}
$$

\section{Scanning Electron Microscope}

Electron microscopy of some bedded sediment samples was performed using an ISI-SS40 scanning electron microscope (SEM) system, manufactured by International Scientific Instruments. Samples were cut perpendicular to bedding with an Isomet low speed saw and the surfaces of the samples were freshly fractured from hammer blows directed perpendicular to bedding on a razor blade (which acted as a high-precision chisel). Samples were glued with epoxy onto $1.5-\mathrm{cm}$-diameter aluminum stubs, placed in an oven at $105^{\circ} \mathrm{C}$ for a few hours to drive off volatiles from the epoxy and water vapor from the sample, and allowed to cool in a desiccator under vacuum. The samples were carbon-coated before being placed into the SEM sample chamber.

\section{SHORE-BASED RESULTS}

Values of $\phi, \rho_{b}$, and $\rho_{d}$, and horizontal and vertical $V_{p}, V_{s}$, and $Q_{p}$, for each sample are listed in Table 1 . Values of horizontal and vertical $\rho^{\prime}$ for each sample at various frequencies are listed in Table 2.

$Q_{p}$ is found to be approximately inversely proportional to $V_{p}$ in these sediments as illustrated in plots of these properties vs. depth (Fig. 2). Resistivity is generally proportional to velocity, and both properties are probably influenced by the degree of matrix cementation. Large amounts of cement increase the bulk modulus and therefore increase $V_{p}$. Because cement closes pore spaces it also acts as a barrier to ionic flow and increases resistivity.

It was difficult to propagate shear waves in these sediments because the sediments where generally not highly lithified. Hence $V_{s}$ values are reported only for six samples. These six samples exhibited a high degree of lithification. With the exception of one lithified sample at 546 mbsf, the shear wave samples came from the bottom of the chalk sequence (795.5830 mbsf).

$V_{p}, V_{s}, Q_{p}$, and $\rho^{\prime}$ were measured horizontally $\left(V_{p h}, V_{s h}\right.$, $Q_{p h}$, and $\left.\rho_{h}^{\prime}\right)$ and vertically $\left(V_{p v}, V_{s v}, Q_{p v}\right.$, and $\left.\rho_{v}^{\prime}\right)$ with respect to the core axis in both bedded and unbedded samples. It was generally found that $V_{p h}>V_{p v}, V_{s h}>V_{s v}$, and $\rho_{v}^{\prime}>\rho_{h}^{\prime}$. Such directional anisotropies are expected in undeformed, horizontal sediments such as these. No generality could be made for $Q_{p}$ anisotropy.

The cause of velocity being higher parallel than perpendicular to bedding has been a subject of debate (e.g., Carlson and Christensen, 1979; Carlson et al., 1983; Kim et al., 1983; Milholland et al., 1980) and is discussed in more detail in a paper in preparation by the authors (see O'Brien et al., 1989). Although $V_{p}$ anisotropy in carbonate sediments has been attributed to preferred orientation of calcite grains (e.g., Milholland et al., 1980), no preferred orientation of calcite was present in any of our samples of calcareous claystone, pelagic chalk, or pelagic limestone, although the samples exhibit a high degree of anisotropy. Anisotropy was found to be primarily influenced by flat pores and to some extent by clay platelets aligned parallel to bedding in the calcareous claystones. However, because the pelagic chalks and limestones lack flat pores, anisotropy is probably controlled only by preferred orientation of clay platelets.

Resistivity is lower in the horizontal direction because it is easier for ions to flow through pores aligned parallel to bedding, suggesting greater permeability in the horizontal rather than the vertical plane. The samples contain some clay that may also cause resistivity to be lower in the horizontal direction. Clay platelets tend to become preferentially oriented parallel to bedding. The excess of negative charge in the outer layer of the clay particle attracts cations from the pore fluid to the clay particle surface. A "double layer" of cations is formed, consisting of a "fixed layer" directly adjacent to the particle surface and a "diffuse layer" that drops off in density exponentially with distance from the fixed layer. The cations of the diffuse layer are free to move under the applied electrical field, which adds to the normal ion flow in the pore space. Thus, the electrical conductivity parallel to the clay platelets is increased (Ward and Fraser, 1967).

Resistivity $\left(\rho^{\prime}\right)$ was also measured at frequencies from 0.1 to $10,000 \mathrm{~Hz}$. Figure 3 shows a plot of mean resistivity vs. frequency for five chalk samples. With the exception of 


\begin{tabular}{|c|c|c|c|c|}
\hline $\begin{array}{l}\text { Core, section, } \\
\text { interval }(\mathrm{cm})\end{array}$ & $\begin{array}{l}\text { Depth } \\
\text { (mbsi) }\end{array}$ & $\begin{array}{c}\text { Vph } \\
(\mathrm{km} / \mathrm{s})\end{array}$ & $\begin{array}{c}V p v \\
(\mathrm{~km} / \mathrm{s})\end{array}$ & $\begin{array}{c}\text { Vp-anisotrop } \\
(\%)\end{array}$ \\
\hline \multicolumn{5}{|l|}{$122-762 \mathrm{C}$ - } \\
\hline $23 \mathrm{X}-2,68-70$ & 371.69 & 1.832 & 1.791 & 2.26 \\
\hline $25 X-3,6-8$ & 391.56 & 1.815 & 1.797 & 1.00 \\
\hline $26 \mathrm{X}-1,28,30$ & 398.29 & 2.023 & 2.008 & 0.74 \\
\hline $27 X-3,58-60$ & 406.09 & 1.968 & 1.891 & 3.99 \\
\hline $30 \mathrm{X}-\mathrm{CC}, 23-25$ & 436.44 & 2.012 & 1.980 & 1.60 \\
\hline $31 X-2,89-91$ & 442.90 & 2.222 & 2.175 & 2.14 \\
\hline $32 X-2,66-69$ & 452.18 & 2.079 & 2.060 & 0.92 \\
\hline $33 X-1,94-96$ & 460.45 & 2.232 & 2.185 & 2.13 \\
\hline $34 X-2,108-110$ & 471.59 & 2.055 & 2.010 & 2.21 \\
\hline $35 \mathrm{X}-1,111-113$ & 479.62 & 2.001 & 1.948 & 2.68 \\
\hline $37 X-3,101-104$ & 501.52 & 1.913 & 1.871 & 2.22 \\
\hline $42 X-1,116-118$ & 546.17 & 2.749 & 2.557 & 7.24 \\
\hline $44 X-3,31-33$ & 567.32 & 2.175 & 2.124 & 2.37 \\
\hline $45 X-1,10-11$ & 573.60 & 2.269 & 2.164 & 4.50 \\
\hline $46 \mathrm{X}-1,38-40$ & 583.39 & 2.333 & 2.232 & 4.42 \\
\hline $47 X-2,77-79$ & 594.78 & 2.243 & 2.168 & 3.40 \\
\hline $48 X-1,43-45$ & 602.44 & 2.310 & 2.194 & 5.15 \\
\hline $49 \times-1,106-109$ & 612.57 & 2.433 & 2.322 & 4.67 \\
\hline $50 X-4,89-91$ & 626.39 & 2.266 & 2.164 & 4.61 \\
\hline $51 X-4,139-141$ & 636.39 & 2.233 & 2.159 & 3.37 \\
\hline $52 X-4,81-83$ & 645.31 & 2.247 & 2.143 & 4.74 \\
\hline $52 X-6,1-3$ & 647.52 & 2.250 & 2.153 & 4.41 \\
\hline $53 X-2,27-29$ & 651.27 & 2.235 & 2.227 & 0.36 \\
\hline $54 X-4,43-45$ & 663.93 & 2.055 & 2.001 & 2.66 \\
\hline $55 X-2,63-65$ & 670.64 & 2.141 & 2.057 & 4.00 \\
\hline $56 \mathrm{X}-3,81-83$ & 681.82 & 2.269 & 2.160 & 4.92 \\
\hline $57 X-2,57-59$ & 689.58 & 2.123 & 2.061 & 2.96 \\
\hline $58 X-3,101-103$ & 701.02 & 2.059 & 2.039 & 0.98 \\
\hline $59 X-2,41-43$ & 708.41 & 2.104 & 2.100 & 0.19 \\
\hline $60 X-1,22-24$ & 716.23 & 2.001 & 1.916 & 4.34 \\
\hline $61 \mathrm{X}-1,31-33$ & 725.82 & 2.161 & 2.069 & 4.35 \\
\hline $62 X-2,123-126$ & 737.74 & 2.328 & 2.239 & 3.90 \\
\hline $64 \mathrm{X}-1,24-26$ & 754.25 & 2.595 & 2.517 & 3.05 \\
\hline $68 X-1,143-145$ & 776.44 & 2.552 & 2.589 & -1.44 \\
\hline $69 \mathrm{X}-1,46-48$ & 780.47 & 2.560 & 2.529 & 1.22 \\
\hline $71 X-1,52-54$ & 790.53 & 2.945 & 2.791 & 5.37 \\
\hline $72 X-1,43-45$ & 795.44 & 2.815 & 2.799 & 0.57 \\
\hline $73 X-2,86-88$ & 801.87 & 2.942 & 2.867 & 2.58 \\
\hline $74 X-2,43-45$ & 806.44 & 3.064 & 2.923 & 4.71 \\
\hline $76 \mathrm{X}-1,54-56$ & 815.05 & 2.769 & 2.576 & 7.22 \\
\hline $77 X-3,125-127$ & 823.76 & 2.716 & 2.576 & 5.29 \\
\hline $78 \mathrm{X}-1,91-93$ & 829.92 & 2.582 & 2.448 & 5.33 \\
\hline $79 X-1,64-66$ & 839.15 & 1.877 & 1.802 & 4.08 \\
\hline $81 X-3,109-111$ & 852.60 & 1.962 & 1.901 & 3.16 \\
\hline $86 \mathrm{X}-2,119-121$ & 885.20 & 1.803 & 1.767 & 2.02 \\
\hline $88 X-6,100-102$ & 910.01 & 1.945 & 1.860 & 4.47 \\
\hline $89 \times-4,28-29$ & 915.78 & 1.942 & 1.858 & 4.42 \\
\hline $90 X-4,88-90$ & 925.88 & 1.957 & 1.850 & 5.62 \\
\hline $91 \mathrm{X}-2,10-12$ & 931.61 & & 1.884 & \\
\hline
\end{tabular}

Vsh
$(\mathrm{km} / \mathrm{s})$

$\underset{(\mathrm{km} / \mathrm{s})}{\mathrm{Vsv}}$

aph

Qpv

Bulk Density
$(\mathrm{g} / \mathrm{cm} 3)$

Grain Dens
$(\mathrm{g} / \mathrm{cm} 3)$

(\%)

931.61

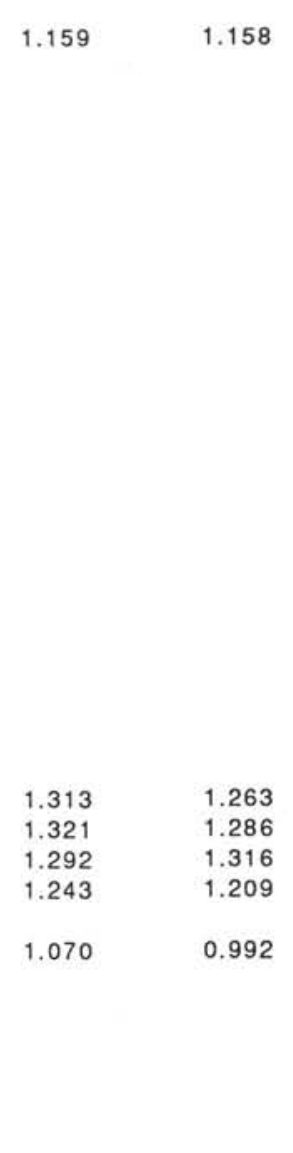
8.7
11.1
9.1
12.9
12.9
8.6
8.2
8.2
8.6
10.3
8.4
9.0
9.0
9.6
8.9
9.2
10.7
10.7
9.0
9.6
10.7
11.0
11.3
7.9
7.7
7.3
6.7
6.5
6.5
9.2
6.7

12.9
13.8
9.7
13.2
13.8
10.2
10.6
9.4
11.0
14.8

1.917

1.953

2.053

1.980
2.080

2.099

2.057
2.075

2.094

2.066

2.092

2.144
2.037

2.001

2.096

2.069

2.101
2.060

2.060
2.123

2.123
2.098

2.098
2.074

2.074

2.074

2.081

1.982

2.086
2.047

1.999

1.999
2.008

2.008
1.951

2.054

2.065
2.149

2.149
2.213

2.213

2.221

2.189

2.189
2.217

2.257

2.194

2.264

2.227

1.647

2.059
2.043

2.147

2.144

2.132

45.8

44.7

38.2

2.700

2.687

2.705

2.706

2.700

2.696

37.2

35.8
37.3

$2.527 \quad 28.6$

$2.693 \quad 32.5$

$\begin{array}{ll}2.704 & 39.3\end{array}$

$\begin{array}{ll}2.773 & 41.7\end{array}$

$\begin{array}{ll}2.696 & 35.5\end{array}$

$\begin{array}{ll}2.703 & 37.4 \\ 2.703 & 35.5\end{array}$

$2.720 \quad 38.5$

$\begin{array}{ll}2.716 & 34.7\end{array}$

$\begin{array}{ll}2.719 & 36.2 \\ 2.705 & 37.1\end{array}$

$\begin{array}{ll}2.721 & 37.7\end{array}$

$2.720 \quad 37.3$

$\begin{array}{ll}2.718 & 43.0\end{array}$

$\begin{array}{ll}2.711 & 36.6 \\ 2.704 & 38.7\end{array}$

$\begin{array}{ll}2.704 & 38.7\end{array}$

$2.722 \quad 41.6$

$2.726 \quad 45.1$

$\begin{array}{ll}2.723 & 39.0 \\ 2.721 & 38.3\end{array}$

$\begin{array}{ll}2.706 & 32.8\end{array}$

$2.721 \quad 29.6$

$\begin{array}{ll}2.723 & 29.2\end{array}$

$\begin{array}{ll}2.720 & 23.0 \\ 2.721 & 31.0\end{array}$

$\begin{array}{ll}2.714 & 28.1\end{array}$

$2.719 \quad 27.0$

$\begin{array}{ll}2.705 & 30.1 \\ 2.705 & 25.9\end{array}$

$\begin{array}{ll}2.705 & 25.9\end{array}$

$\begin{array}{ll}2.396 & 53.9\end{array}$

$\begin{array}{ll}2.678 & 37.0\end{array}$

$2.694 \quad 38.5$

$\begin{array}{ll}2.756 & 34.8 \\ 2.748 & 34.7\end{array}$

$2.702 \quad 33.6$ 
Table 2. Shore-based electrical resistivity values in horizontal and vertical directions at frequencies of $0.1,1.0,10,100,1000$, and $10000 \mathrm{~Hz}$ for samples from $\mathrm{Hole} 762 \mathrm{C}$.

\begin{tabular}{|c|c|c|c|c|c|c|c|c|c|c|c|c|c|}
\hline $\begin{array}{l}\text { Core, section, } \\
\text { interval }(\mathrm{cm})\end{array}$ & $\begin{array}{l}\text { Depth } \\
\text { (mbsf) }\end{array}$ & $\begin{array}{l}\text { Horizontal } \\
\text { at } 0.1 \mathrm{~Hz} \\
\text { (ohm-m) }\end{array}$ & $\begin{array}{l}\text { Vertical } \\
\text { at } 0.1 \mathrm{~Hz} \\
\text { (ohm-m) }\end{array}$ & $\begin{array}{l}\text { Horizontal } \\
\text { at } 1 \mathrm{~Hz} \\
\text { (ohm-m) }\end{array}$ & $\begin{array}{l}\text { Vertical } \\
\text { at } 1 \mathrm{~Hz} \\
(\mathrm{ohm}-\mathrm{m})\end{array}$ & $\begin{array}{l}\text { Horizontal } \\
\text { at } 10 \mathrm{~Hz} \\
(\mathrm{ohm}-\mathrm{m})\end{array}$ & $\begin{array}{l}\text { Vertical } \\
\text { at } 10 \mathrm{~Hz} \\
(\mathrm{ohm}-\mathrm{m})\end{array}$ & $\begin{array}{l}\text { Horizontal } \\
\text { at } 100 \mathrm{~Hz} \\
(\mathrm{ohm}-\mathrm{m})\end{array}$ & $\begin{array}{l}\text { Vertical } \\
\text { at } 100 \mathrm{~Hz} \\
\text { (ohm-m) }\end{array}$ & $\begin{array}{l}\text { Horizontal } \\
\text { at } 1,000 \mathrm{~Hz} \\
(0 \mathrm{hm}-\mathrm{m})\end{array}$ & $\begin{array}{c}\text { Vertical } \\
\text { at } 1,000 \mathrm{~Hz} \\
(0 \mathrm{hm}-\mathrm{m})\end{array}$ & $\begin{array}{l}\text { Horizontal } \\
\text { at } 10,000 \mathrm{~Hz} \\
(0 \mathrm{hm}-\mathrm{m})\end{array}$ & $\begin{array}{l}\text { Vertical } \\
\text { at } 10,000 \mathrm{~Hz} \\
(\mathrm{ohm}-\mathrm{m})\end{array}$ \\
\hline \multicolumn{14}{|l|}{$122-762 \mathrm{C}$. } \\
\hline $23 X-2,68-70$ & 371.69 & 1.041 & 1.292 & 0.897 & 1.155 & 0.875 & 1.134 & 0.826 & 1.088 & 0.795 & 1.057 & 0.805 & 1.077 \\
\hline $25 X-3,6-8$ & 391.56 & 1.047 & 1.426 & 0.936 & 1.378 & 0.913 & 1.354 & 0.863 & 1.310 & 0.833 & 1.288 & 0.847 & 1.319 \\
\hline $26 \mathrm{X}-1,28-31$ & 398.29 & 1.271 & 1.566 & 1.207 & 1.513 & 1.180 & 1.485 & 1.130 & 1.430 & 1.101 & 1.392 & 1.122 & 1.413 \\
\hline $27 X-3,58-60$ & 406.09 & 1.205 & 1.621 & 1.137 & 1.594 & 1.112 & 1.569 & 1.062 & 1.533 & 1.033 & 1.520 & 1.051 & 1.563 \\
\hline $30 X-C C, 23-25$ & 436.44 & 1.265 & 1.640 & 1.183 & 1.583 & 1.156 & 1.556 & 1.107 & 1.511 & 1.080 & 1.486 & 1.103 & 1.518 \\
\hline $31 X-2,89-91$ & 442.90 & 1.222 & 1.409 & 1.183 & 1.370 & 1.154 & 1.342 & 1.104 & 1.289 & 1.078 & 1.260 & 1.098 & 1.288 \\
\hline $32 X-2,66-69$ & 452.18 & 1.089 & 1.273 & 1.027 & 1.230 & 1.002 & 1.201 & 0.953 & 1.150 & 0.928 & 1.123 & 0.944 & 1.139 \\
\hline $33 \mathrm{X}-1,94-96$ & 460.45 & 1.238 & 1.405 & 1.193 & 1.353 & 1.158 & 1.319 & 1.102 & 1.262 & 1.073 & 1.229 & 1.089 & 1.244 \\
\hline $34 \mathrm{X}-2,108-110$ & 471.59 & 1.214 & 1.493 & 1.187 & 1.479 & 1.162 & 1.448 & 1.116 & 1.396 & 1.092 & 1.351 & 1.114 & 1.363 \\
\hline $35 \mathrm{X}-1,111-113$ & 479.62 & 1.221 & 1.735 & 1.149 & 1.701 & 1.116 & 1.659 & 1.061 & 1.589 & 1.034 & 1.548 & 1.047 & 1.565 \\
\hline $42 X-1,116-118$ & 546.17 & 3.557 & 4.825 & 3.496 & 4.733 & 3.336 & 4.577 & 3.139 & 4.369 & 3.065 & 4.260 & 3.235 & 4.475 \\
\hline $44 X-3,31-33$ & 567.32 & 2.626 & 3.398 & 2.591 & 3.348 & 2.413 & 3.152 & 2.213 & 2.936 & 2.119 & 2.830 & 2.209 & 2.950 \\
\hline $45 \mathrm{X}-1,10-11$ & 573.60 & 2.466 & 2.960 & 2.362 & 2.880 & 2.165 & 2.673 & 1.972 & 2.462 & 1.838 & 2.260 & 1.900 & 2.289 \\
\hline $46 \mathrm{X}-1,38-40$ & 583.39 & 2.267 & 2.200 & 2.128 & 2.131 & 1.907 & 1.900 & 1.730 & 1.718 & 1.630 & 1.640 & 1.680 & 1.708 \\
\hline $47 X-2,77-79$ & 594.78 & 3.134 & 3.442 & 3.013 & 3.372 & 2.785 & 3.103 & 2.587 & 2.906 & 2.438 & 2.788 & 2.487 & 2.893 \\
\hline $49 X-1,106-109$ & 612.57 & 3.204 & 3.702 & 3.121 & 3.574 & 2.880 & 3.299 & 2.674 & 3.093 & 2.518 & 2.881 & 2.567 & 2.950 \\
\hline $50 X-4,89-91$ & 626.93 & 2.122 & 2.503 & 2.050 & 2.372 & 2.017 & 2.327 & 1.932 & 2.222 & 1.872 & 2.150 & 1.986 & 2.278 \\
\hline $51 X-4,139-141$ & 636.39 & 2.624 & 3.120 & 2.527 & 3.091 & 2.477 & 3.033 & 2.346 & 2.883 & 2.243 & 2.778 & 2.361 & 2.928 \\
\hline $52 X-4,81-83$ & 645.31 & 2.399 & 2.991 & 2.319 & 2.879 & 2.269 & 2.814 & 2.135 & 2.657 & 2.054 & 2.555 & 2.167 & 2.694 \\
\hline $52 X-6,1-3$ & 647.52 & 3.029 & 3.089 & 2.966 & 3.007 & 2.696 & 2.722 & 2.508 & 2.533 & 2.308 & 2.440 & 2.328 & 2.551 \\
\hline $53 X-2, \quad 27-29$ & 651.27 & 2.260 & 2.642 & 2.253 & 2.602 & 2.186 & 2.524 & 2.031 & 2.379 & 1.941 & 2.316 & 2.044 & 2.457 \\
\hline $54 X-4,43-45$ & 663.93 & 2.261 & 2.584 & 2.206 & 2.626 & 2.084 & 2.502 & 1.902 & 2.312 & 1.816 & 2.220 & 1.907 & 2.333 \\
\hline $55 X-2,63-65$ & 670.64 & 2.128 & 2.546 & 2.046 & 2.478 & 1.748 & 2.189 & 1.570 & 2.007 & 1.487 & 1.859 & 1.550 & 1.878 \\
\hline $56 X-3,81-83$ & 681.82 & 2.821 & 2.903 & 2.688 & 2.822 & 2.396 & 2.523 & 2.220 & 2.344 & 2.084 & 2.218 & 2.135 & 2.305 \\
\hline $57 X-2,57-59$ & 689.58 & 2.982 & 3.369 & 2.886 & 3.251 & 2.601 & 2.956 & 2.400 & 2.738 & 2.136 & 2.442 & 2.133 & 2.429 \\
\hline $58 X-3,101-103$ & 701.02 & 1.546 & 1.672 & 1.431 & 1.622 & 1.397 & 1.586 & 1.337 & 1.487 & 1.308 & 1.419 & 1.391 & 1.500 \\
\hline $59 X-2,41-43$ & 708.41 & 1.809 & 1.976 & 1.825 & 1.929 & 1.700 & 1.795 & 1.512 & 1.602 & 1.431 & 1.522 & 1.510 & 1.612 \\
\hline $60 X-1,22-24$ & 716.23 & 1.483 & 1.515 & 1.420 & 1.456 & 1.384 & 1.413 & 1.273 & 1.302 & 1.181 & 1.223 & 1.235 & 1.278 \\
\hline $61 X-1,31-33$ & 725.82 & 1.649 & 1.689 & 1.626 & 1.736 & 1.576 & 1.706 & 1.451 & 1.574 & 1.385 & 1.479 & 1.461 & 1.554 \\
\hline $62 X-2,123-126$ & 737.74 & 1.935 & 2.193 & 1.878 & 2.155 & 1.861 & 2.113 & 1.743 & 1.954 & 1.662 & 1.843 & 1.747 & 1.928 \\
\hline $68 \mathrm{X}-1,143-145$ & 776.44 & 2.755 & 2.807 & 2.728 & 2.760 & 2.686 & 2.709 & 2.549 & 2.586 & 2.473 & 2.536 & 2.610 & 2.688 \\
\hline $69 X-1,46-48$ & 780.47 & 3.109 & 3.069 & 3.095 & 3.151 & 3.012 & 3.029 & 2.860 & 2.853 & 2.710 & 2.754 & 2.808 & 2.884 \\
\hline $71 X-1,52-54$ & 790.53 & 3.600 & 4.368 & 3.777 & 4.287 & 3.673 & 4.174 & 3.506 & 3.999 & 3.426 & 3.908 & 3.600 & 4.122 \\
\hline $72 X-1,43-45$ & 795.44 & 2.637 & 2.923 & 2.696 & 2.926 & 2.561 & 2.804 & 2.391 & 2.642 & 2.305 & 2.537 & 2.421 & 2.648 \\
\hline $73 X-2,86-88$ & 801.87 & 3.409 & 3.154 & 3.567 & 3.181 & 3.395 & 3.046 & 3.193 & 2.899 & 3.022 & 2.823 & 3.123 & 2.969 \\
\hline $74 X-2,43-45$ & 806.44 & 3.622 & 3.703 & 3.670 & 3.726 & 3.474 & 3.602 & 3.286 & 3.450 & 3.125 & 3.330 & 3.248 & 3.480 \\
\hline $76 X-1,54-56$ & 815.05 & 2.690 & 3.215 & 2.632 & 3.111 & 2.603 & 3.069 & 2.469 & 2.942 & 2.389 & 2.875 & 2.517 & 3.047 \\
\hline $77 X-3,125-127$ & 823.76 & 2.932 & 3.716 & 2.872 & 3.673 & 2.844 & 3.603 & 2.716 & 3.442 & 2.646 & 3.367 & 2.795 & 3.568 \\
\hline $78 X-1,91-93$ & 829.92 & 2.879 & 3.422 & 2.902 & 3.420 & 2.825 & 3.325 & 2.649 & 3.135 & 2.529 & 3.002 & 2.642 & 3.138 \\
\hline $79 \mathrm{X}-1,64-66$ & 839.15 & 1.793 & 1.646 & 1.745 & 1.597 & 1.622 & 1.506 & 1.410 & 1.353 & 1.248 & 1.257 & 1.219 & 1.295 \\
\hline $86 X-2,119-121$ & 885.20 & 2.187 & 2.739 & 2.177 & 2.698 & 2.062 & 2.589 & 1.865 & 2.403 & 1.665 & 2.286 & 1.675 & 2.358 \\
\hline $88 X-6,100-102$ & 910.01 & 2.208 & 3.317 & 2.212 & 3.267 & 2.084 & 3.131 & 1.914 & 2.957 & 1.769 & 2.791 & 1.786 & 2.866 \\
\hline $89 X-4,28-29$ & 915.78 & 2.164 & 3.365 & 2.156 & 3.350 & 2.007 & 3.186 & 1.828 & 2.994 & 1.706 & 2.816 & 1.742 & 2.856 \\
\hline $90 X-4,88-89$ & 925.88 & 2.273 & 3.323 & 2.249 & 3.179 & 2.148 & 3.021 & 1.986 & 2.852 & 1.844 & 2.757 & 1.892 & 2.893 \\
\hline
\end{tabular}



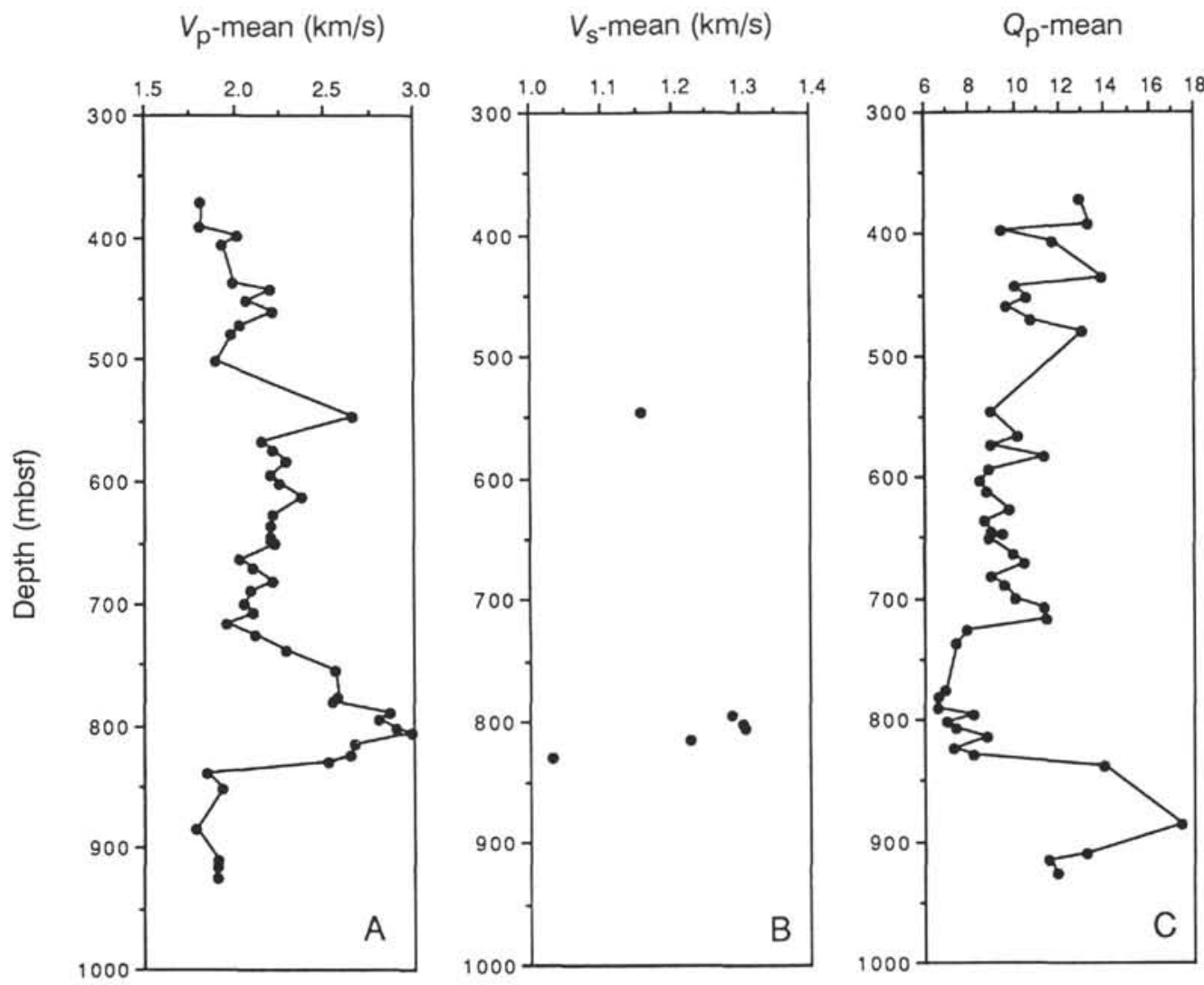

Mean resistivity (ohm-m)

Figure 2. Physical properties of Hole $762 \mathrm{C}$ plotted against depth. A. Mean compressional velocity. B. Mean shear velocity. C. Mean compressional $Q$. D. Mean resistivity at $100 \mathrm{~Hz}$.

Sample $122-762 \mathrm{C}-74 \mathrm{X}-2,43-45 \mathrm{~cm}$, values of $\rho^{\prime}$ at $1.0 \mathrm{~Hz}$ are slightly less than those at $0.1 \mathrm{~Hz}$. Resistivity decreases more rapidly from 1.0 to $1,000 \mathrm{~Hz}$, and increases between 1,000 and $10,000 \mathrm{~Hz}$. Such a trend was observed by Katsube $(1977$, his fig. 1.4) for higher $\rho^{\prime}$ rocks $(50-5000$ ohm-meters [ohm-m]), but was offset slightly toward lower frequencies. However, Katsube's data suggest that the trend shifts to higher frequencies in the case of low $\rho^{\prime}$ rocks, such as marine sediments $\left(\rho^{\prime}\right.$ $=0.5-5.0 \mathrm{ohm}-\mathrm{m})$. This trend of changing $\rho^{\prime}$ with frequency is probably due to "electrode polarization" (Ward and Fraser, 1967; T. J. Katsube, pers. comm., 1989). Above $10,000 \mathrm{~Hz}$, charge is transferred between the electrodes and pore fluid by charging and discharging of the electrode's "double layer," as in a capacitor. This effect is frequency-independent. Between about 10,000 and $0.01 \mathrm{~Hz}$, current is also transmitted across the electrode-pore fluid interface as previously described, but the current is also transmitted physically by an electrochemical reaction, such as the conversion of an atom to an ion or vice versa. This process is frequency-dependent, and more charge is transmitted by this method as frequency decreases. Below about $0.01 \mathrm{~Hz}$, charge is primarily transmitted electrochemically, and the process becomes frequency dependent.

\section{COMPARISON WITH SHIPBOARD DATA}

Values of shipboard and shore-based laboratory $V_{p}$ as a function of depth are illustrated in Figure 4A. The two sets of $V_{p}$ values agree well between 300 and 670 mbsf. From 670 to $820 \mathrm{mbsf}$, the shore measurements are much greater. The measurements again coincide from 820 mbsf to the bottom of the hole. This discrepancy is due to a calibration error in the shipboard Hamilton Frame velocimeter system. The system is calibrated every 1 to 2 days in order to determine a "system delay time" caused by the electronics and the thickness of the epoxy covering the transducers. This system delay must be subtracted from the traveltimes of acoustic waves traveling through the sediment samples before velocities are calculated. A value of approximately 2.75 microseconds $(\mu \mathrm{s})$ was used as a system delay for most of Hole $762 \mathrm{C}$. However, between 670 and $820 \mathrm{mbsf}$, a calibration run resulted in a value of $-0.01 \mu \mathrm{s}$ for the system delay. A calibration run the next day resulted in a value near $2.75 \mu \mathrm{s}$, which is why the shipboard and shore-based measurements once again agree below $820 \mathrm{mbsf}$. The system delay used between 670 and 820 mbsf was not recognized as erroneous during the cruise.

The sonic $\log V_{p}$ data for Hole $762 \mathrm{C}$ are compared to the shore-based data in Figure 4B. The log data are generally 0.3 $\mathrm{km} / \mathrm{s}$ higher than the shore-based data. The elevated log velocities are most likely due to in-situ overburden pressure because velocities of sediment samples have been shown to increase with differential pressure in laboratory measurements (e.g., Kim et al., 1983). This difference was subtracted from the log data in Figure 4 in order to compare the velocity trends of both data sets. The velocity trends seem to match up well, even in the depth range where the Hamilton Frame velocities do not agree with the shore-based velocities. This matching of trends supports the supposition that the shipboard calibration problem was responsible for the divergence of the Hamilton Frame and shore-based velocity data sets.

There is good agreement between shipboard and shorebased grain density $\left(\rho_{g}\right)$ values (Fig. 5A), though there appears to be more scatter in the shipboard data. This scatter may be due to the method used to determine shipboard volumes. A helium-displacement pycnometer was used to determine wet and dry sample volumes, which were used together with wet 


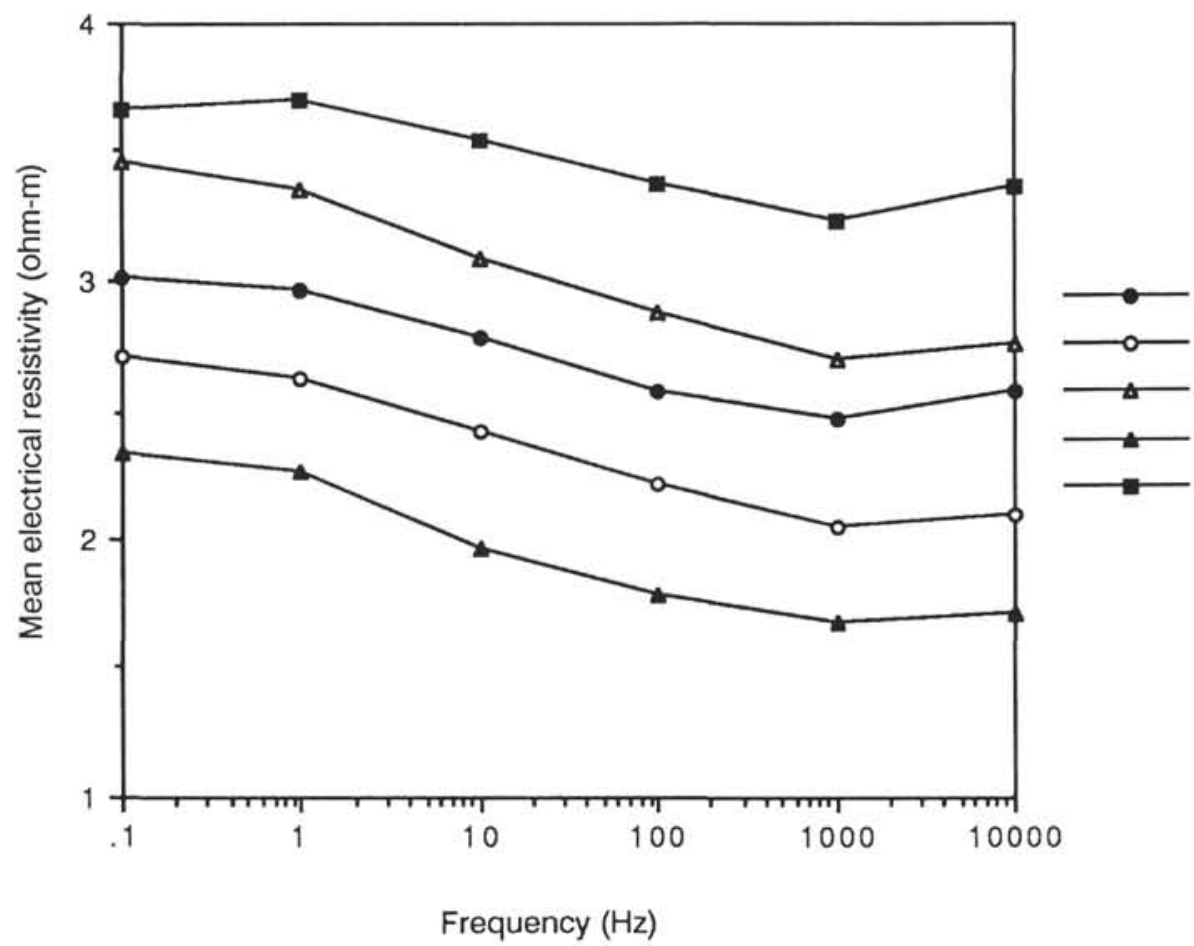

Sample 122-762C-44X-3, $31-33 \mathrm{~cm}$

Sample 122-762C-45X-1, 10-11 cm

Sample 122-762C-49X-1, $106-109 \mathrm{~cm}$

Sample 122-762C-55X-2, 63-65 cm

Sample 122-762C-74X-2, $43-45 \mathrm{~cm}$

Figure 3. Electrical resistivity vs. frequency for five chalk samples from Hole $762 \mathrm{C}$.

and dry weights to calculate $\rho_{d}, \rho_{g}$, and $\phi$. Usually three or four successive volume measurements were made using the pycnometer, and the average value was chosen as the appropriate volume of the sample. The dry volumes produced a range in which the maximum and minimum values often differed by about $0.05 \mathrm{~cm}^{3}$. This range is about two orders of magnitude greater than the range of volumes produced in successive shore-based dry volume measurements. Therefore, more scatter in shipboard density measurements would be expected.

A plot of shipboard vs. shore-based grain densities (Fig. 6) shows that most of the data fall in a single cluster. The larger scatter in shipboard data is evident. A line with the equation $Y=X$ passes through the cluster's center, which corresponds to the grain density of calcite $\left(2.71 \mathrm{~g} / \mathrm{cm}^{3}\right)$. There is also good agreement between shipboard and shorebased porosity $(\phi)$ data (Fig. 5B). A plot of shipboard vs. shore-based porosities (Fig. 7) shows a linear trend. The $Y=$ $X$ line passes through the center of this cluster of data points.

Shipboard bulk density values, however, are consistently higher than shore-based data (Fig. 5C). A plot of shipboard vs. shore-based bulk densities (Fig. 8) shows a relatively linear cluster of data points, but the cluster lies below the $Y$ $=X$ line. A linear regression yields the equation $Y=$ $0.7032 X+0.5608$. This discrepancy between the shipboard and shore-based measurements is again due to the shipboard method of measuring volumes with a helium-displacement pycnometer. The pycnometer's manufacturer, Quantachrome, has stated that the machine was not designed to measure wet samples (David Seltzer, pers. comm., 1988). Moisture in the sample chamber causes an erroneously high pressure reading (also observed in a smaller Quantachrome helium-displacement pycnometer used in our Hawaii laboratory), resulting in a volume that is too low and a calculated bulk density that is therefore too high.
When a sample is freeze-dried aboard ship, only water is lost. Because the density of water is about $1.0 \mathrm{~g} / \mathrm{cm}^{3}$, the difference between the wet and dry sample volumes should be equal to the difference between the wet and dry sample weights, or

wet weight - dry weight $=$ wet volume - dry volume.

However, what is actually observed aboard ship is

wet weight - dry weight $>$ wet volume - dry volume.

This relation was also observed during ODP Leg 110 (Roy Wilkens, pers. comm., 1989; Wilkens et al., 1990), and the cause is the low volume obtained from the pycnometer for wet samples. This problem becomes even more significant when samples of low porosity, such as cherts and basalts, are measured. Because these samples contain only a small amount of water, wet volumes of such samples should be just slightly greater than their dry volumes. But as a result of the pycnometer determining wet volumes that are too small, shipboard wet volumes for cherts were actually smaller than the dry volumes for the same samples, which made it appear as if the samples gained volume upon drying even though they lost mass! When the difference between wet and dry volumes is large, the wet volume error is not apparent.

Pycnometer volume measurements seem to be accurate for dry samples. Therefore, an alternate method for determining shipboard wet volumes for bulk density is to add the difference between the wet and dry weights to the dry sample volume, or

wet volume $=$ dry volume + (wet weight - dry weight $).$

This equation is derived from equation 8. Again, the calculation uses a density of $1.0 \mathrm{~g} / \mathrm{cm}^{3}$ for the water lost from 


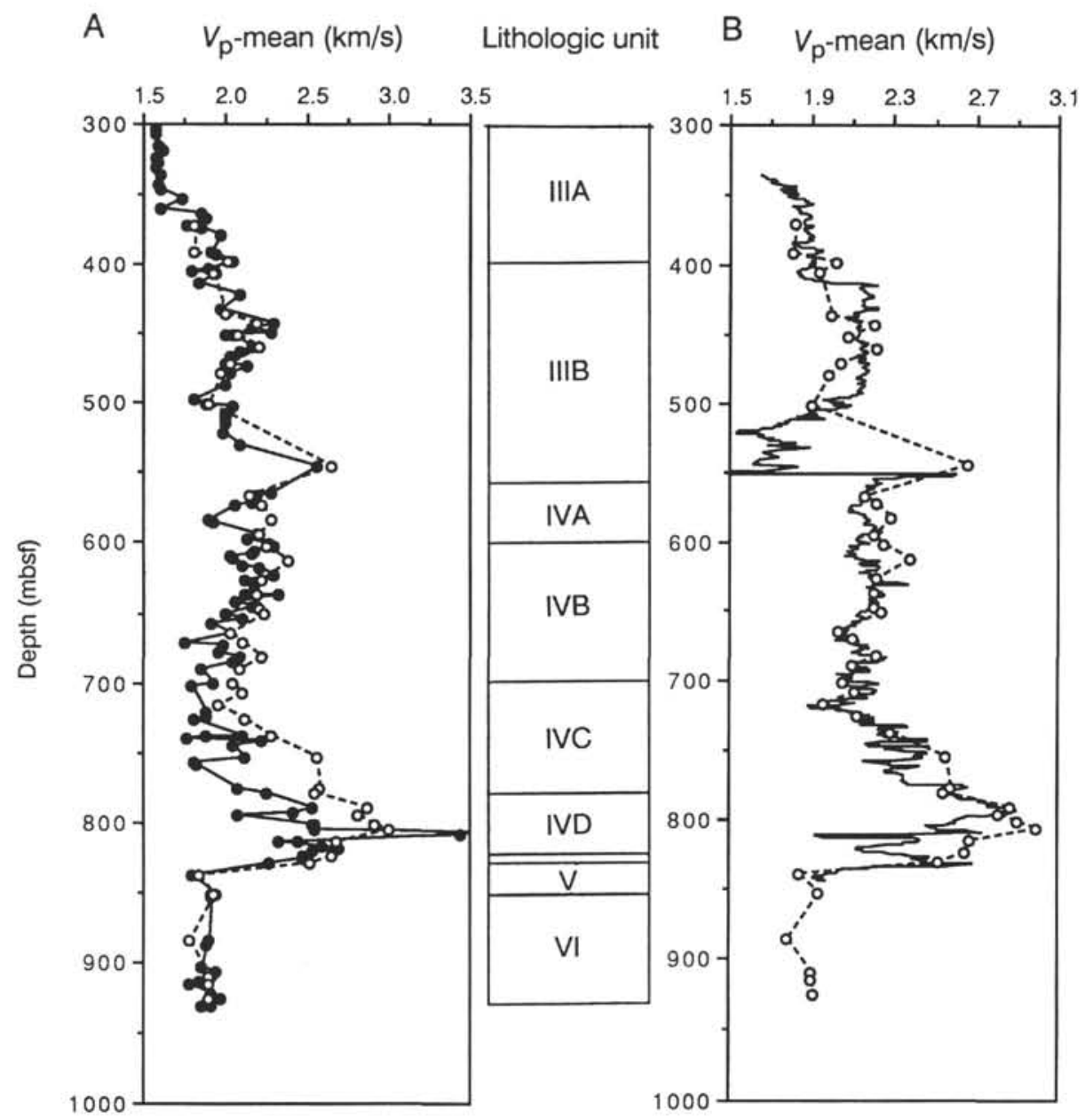

Figure 4. A. Mean shipboard and shore-based compressional velocity $\left(V_{p}\right)$ vs. depth for samples from Hole $762 \mathrm{C}$. Shipboard measurements are indicated by solid line and solid circles, shore-based measurements by dashed line and open circles. Measurements diverge in the range 670-820 mbsf. B. Sonic $\log$ and mean shore-based $V_{p}$ vs. depth for samples from Hole $762 \mathrm{C}$. Sonic $\log$ measurements are indicated by solid line, shore-based measurements by dashed line and open circles. Shore-based measurements coincide well with log velocities, especially in the depth range where shore-based and shipboard data diverge. Lithologic units are indicated. Units III and IV are nannofossil chalk, Unit V is calcareous claystone, and Unit VI is claystone.

the sample upon freeze-drying. The wet volumes for shipboard samples used for Figure 8 were recalculated using equation 10 and the shipboard bulk densities were recalculated using the new wet volumes. The recalculated bulk densities are plotted vs. shore-based bulk densities in Figure 9. A line with the equation $Y=X$ passes through the center of this cluster of data points. Therefore, calculating wet volumes using equation 10 results in shipboard bulk densities that are comparable to shore-based results. We recommend that ODP routinely use equation 10 instead of using wet sample volumes obtained from the pycnometer.

\section{POROSITY-FORMATION FACTOR SYSTEMATICS}

The electrical resistivity of marine sediments changes with depth due to various lithologic parameters, such as pore tortuosity (i.e., the length of the ionic path relative to the length of the sample), the size of the pores, and the permeability of the sediments. These parameters are largely affected by the amount of cementation of the grain matrix. Sediment samples of similar pore structures but different grain types (e.g., clay vs. biogenic carbonate) may have different resistivities as well. Resistivity can also be affected by the salinity of the pore water, so if there is a salinity gradient at a particular drill site, the resistivity will change due to nonlithologic factors. It is, therefore, often convenient to normalize electrical resistivity by expressing it in terms of the formation factor $(F)$, which is defined as

$$
F=\rho 0 / \rho_{\mathrm{w}},
$$

where $\rho_{0}$ is the electrical resistivity of the saturated sediment, and $\rho_{w}$ is the electrical resistivity of the pore water.

In calculating $F$, the electrical resistivity of seawater is taken as 0.2008 ohm-m assuming a salinity of 35 ppt (a 0.60 -molar concentration of $\mathrm{NaCl}$ ) (Weast and Astle, 1981). The formation factor $(F)$ generally increases with decreasing porosity $(\phi)$. Archie (1942) showed that these two parameters can be related empirically by the equation

$$
F=\phi^{-m},
$$

where $\phi$ is fractional porosity and $m$ is called the cementation factor. This equation is known as Archie's Law, and was 


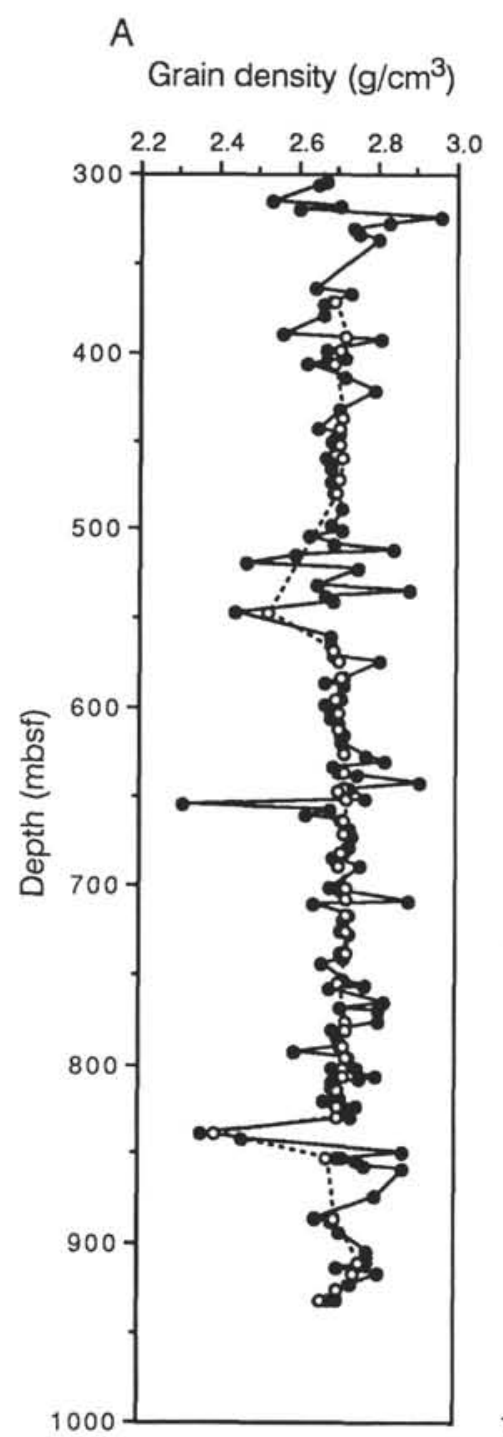

B Porosity (\%)

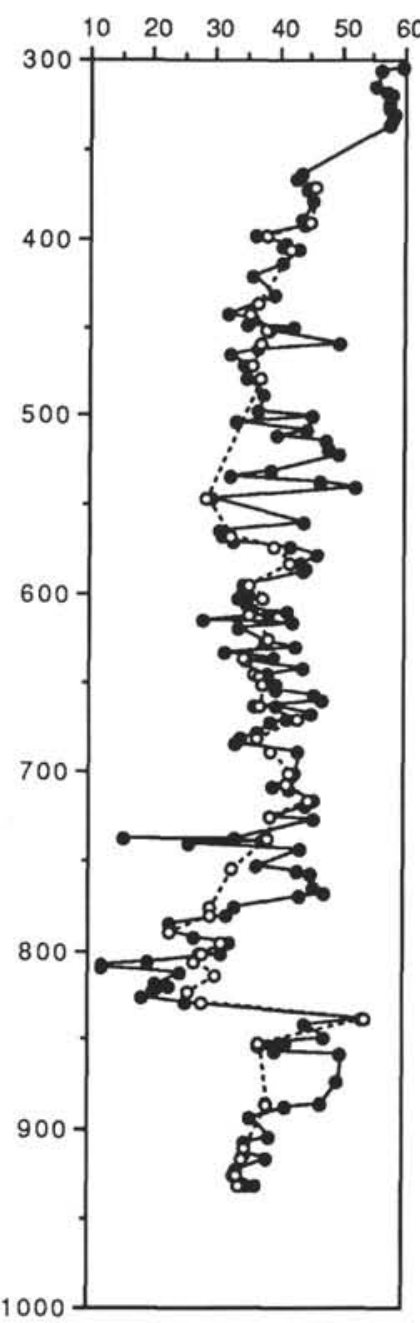

C

Bulk density $\left(\mathrm{g} / \mathrm{cm}^{3}\right)$

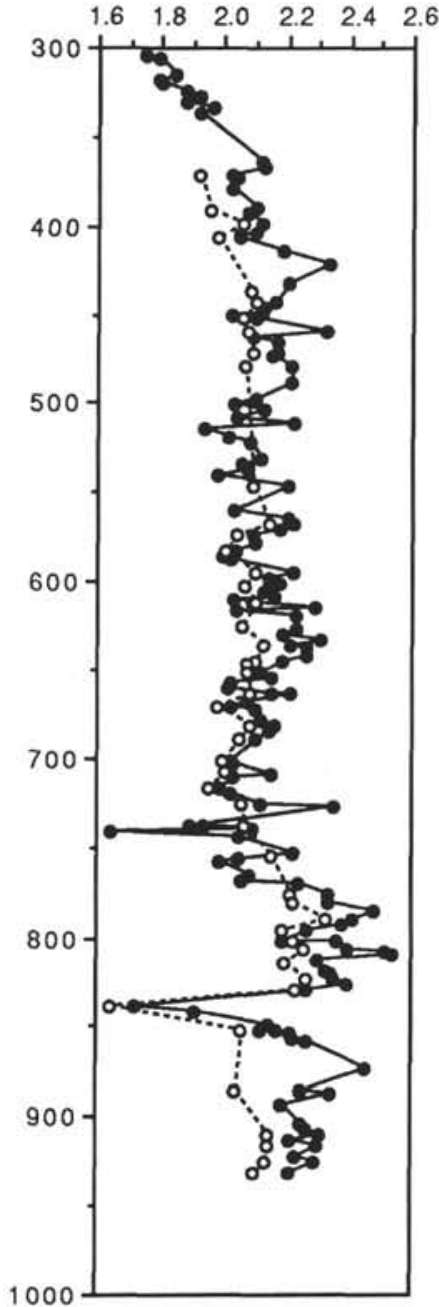

Figure 5. Shipboard and shore-based index properties vs. depth for Hole $762 \mathrm{C}$. Shipboard measurements are indicated by solid line and solid circles, shore-based measurements by dashed line and open circles. A. Grain density. B. Porosity. C. Bulk density.

modified by Winsauer et al. (1952) as an empirical formula for various sandstones,

$$
F=A \phi^{-m},
$$

where $A$ is a constant. This is known as the Winsauer equation. The cementation factor for all rocks and sediments is a number that is generally between 1.3 and 2.3 . The value of $m$ increases with increasing cementation of the grain matrix. It has been shown that $m=1.4$ for slightly consolidated sandstones, $m=1.7$ for consolidated sandstones, and $m=2.0$ for limestones and dolomites (Ward and Frazer, 1967). The value of $m$ can be determined for a given data set by taking the log of both sides of equation 13 , plotting $\log \phi$ vs. $\log F$, and determining a linear regression of the data, which would have the equation

$$
\log F=\log A-m \log \phi .
$$

These plots have been made for the chalks from Units III and IV (Fig. 10) and for the claystones from Units V and IV
(Fig. 11) from Hole 762C. The cementation factor for the chalks is 1.81 , predicting (correctly) that these sediments are well lithified. The claystone samples are quite friable, which is probably why the cementation factor is a low 1.24 .

In the $\log \phi$ vs. $\log F$ plots, $\log A$ is the $y$-intercept. Therefore, the constant $A$ represents the empirically determined formation factor at $100 \%$ porosity. The values of $A$ for the chalks and mudstones (1.52 and 3.21, respectively) are greater than 1.0, indicating a resistivity greater than that of seawater. These high values of $A$ suggest that the Winsaurer equation does not hold for sediments with high porosities.

The values of $m$ are based on formation factors that were calculated from average electrical resistivities of the horizontal and vertical measurements at $100 \mathrm{~Hz}$. Calculations were made from the resistivity data of the chalk samples to determine the range of $m$ values that are possible based on direction and frequency of measurement. Resistivity was shown to vary with frequency in Figure 3. Resistivities are low at $1000 \mathrm{~Hz}$. At this frequency, $m=1.92$ for horizontal measurements and 1.78 for vertical measurements. Resistivities are high at 1.0 


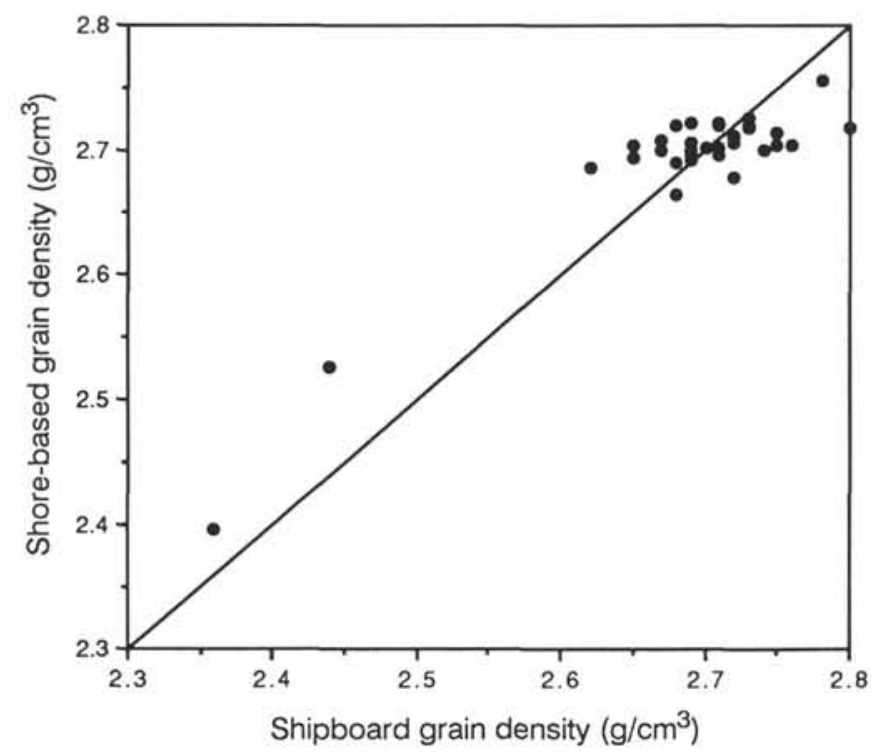

Figure 6. Shipboard vs. shore-based grain density. Line indicates the relation $Y=X$ and is not a linear regression of the data. Line passes through the center of the cluster of data points at the value of 2.71 $\mathrm{g} / \mathrm{cm}^{2}$, the density of calcite. Cluster shows slightly more scatter in the $X$ direction (the shipboard data).

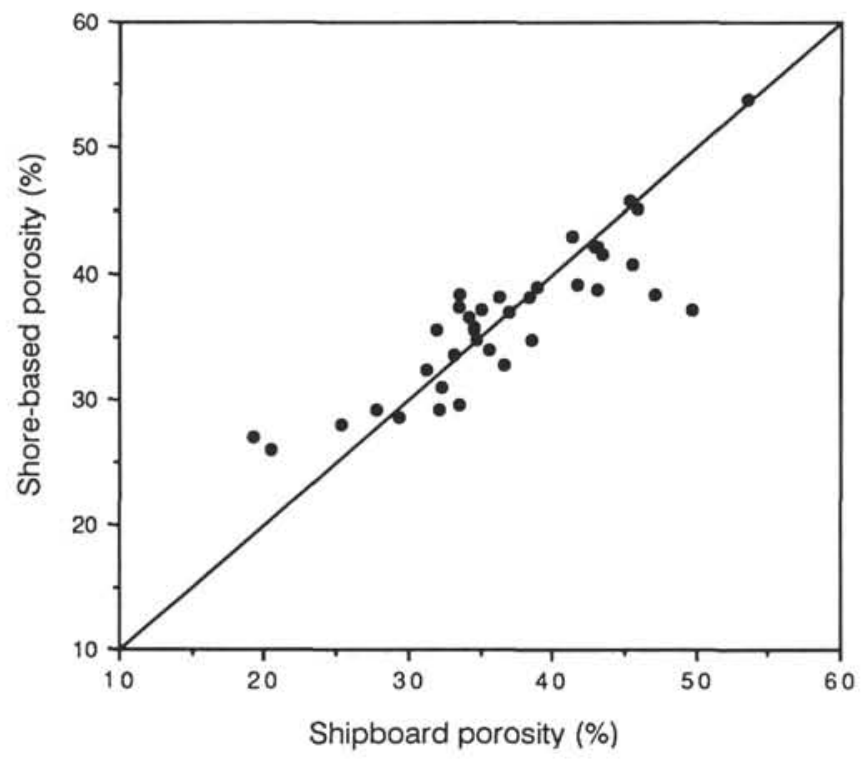

Figure 7. Shipboard vs. shore-based porosity. Line indicates the relation $Y=X$ and is not a linear regression of the data. Line passes through the center of the linear cluster of data points.

$\mathrm{Hz}$. At this frequency, $m=1.83$ for horizontal measurements and 1.67 for vertical measurements. Therefore, $m$ for these chalks can vary from 1.67 to 1.92 depending upon direction and frequency.

\section{VELOCITY GRADIENTS}

Velocity gradients are calculated by dividing an interval velocity by the depth interval, which in essence is the slope of a velocity vs. depth plot. The velocity data from Site 762 exhibit unusual gradients for calcareous sediments. Hamilton (1980) reports that gradients for calcareous sediments from the

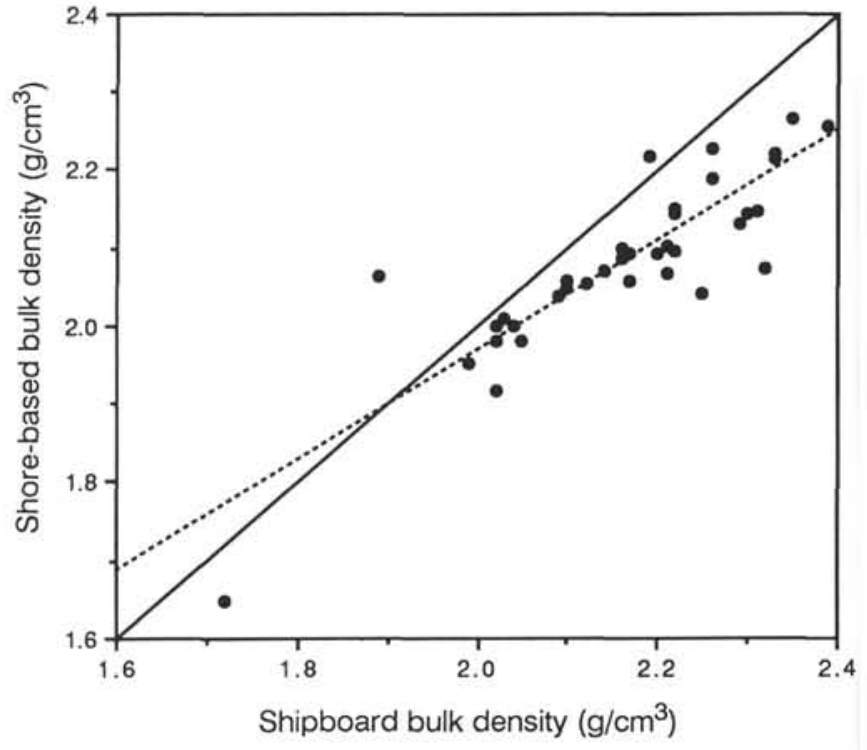

Figure 8. Shipboard vs. shore-based bulk density. Solid line indicates the relation $Y=X$. Dashed line is the linear regression of the data and has the equation $Y=0.7032 X+0.5608$. Shipboard bulk densities are consistently higher than shore-based data because shipboard bulk densities incorporate volumes determined from wet samples in a helium-displacement pycnometer. Pycnometer volume data from wet samples are systematically too low, which result in bulk densities that are too high.

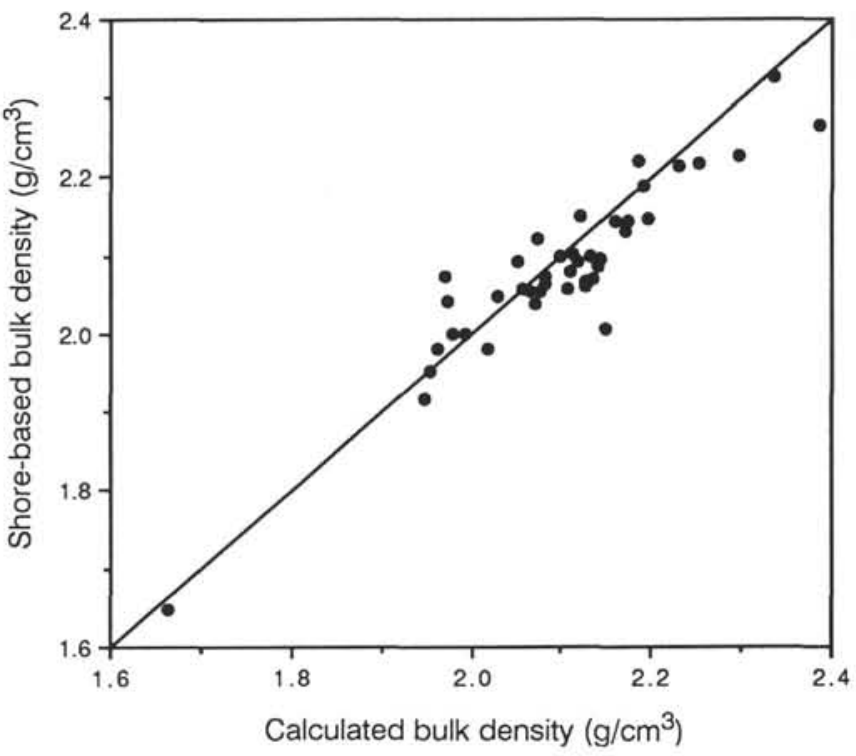

Figure 9. Recalculated shipboard bulk density vs. shore-based data. Shipboard bulk densities were recalculated using wet volumes determined from equation 10 in the text. Line indicates the relation $Y=X$ and is not a linear regression of the data. Line passes through the center of the linear cluster of data points.

Ontong-Java Plateau typically range between 1.3 to $1.9 \mathrm{~km} /$ $\mathrm{s} / \mathrm{km}$ (or " $\mathrm{s}^{-1}$ ") between 0 and $1000 \mathrm{mbsf}$, and that the largest gradients are toward the top of the hole and steadily decrease with depth. However, the gradient for about the first $350 \mathrm{~m}$ of Site 762 is only $0.18 \mathrm{~s}^{-1}$. Within this relatively large depth range (which is composed almost entirely of nannofossil 


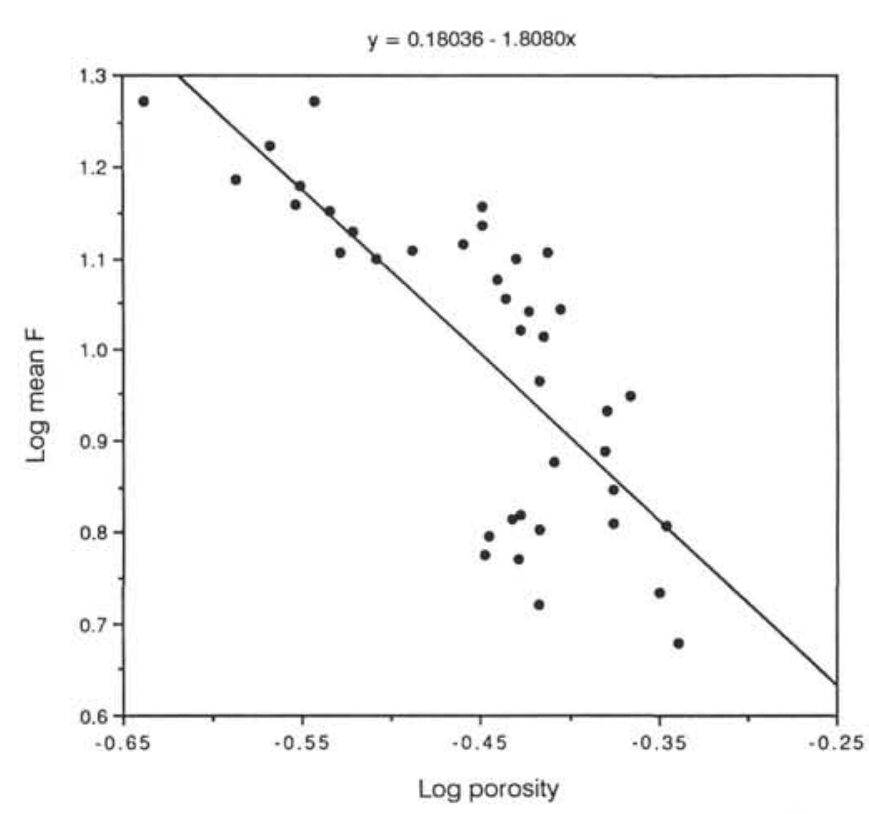

Figure 10. Plot of $\log$ porosity vs. $\log$ mean formation factor for chalks from Units III and IV.

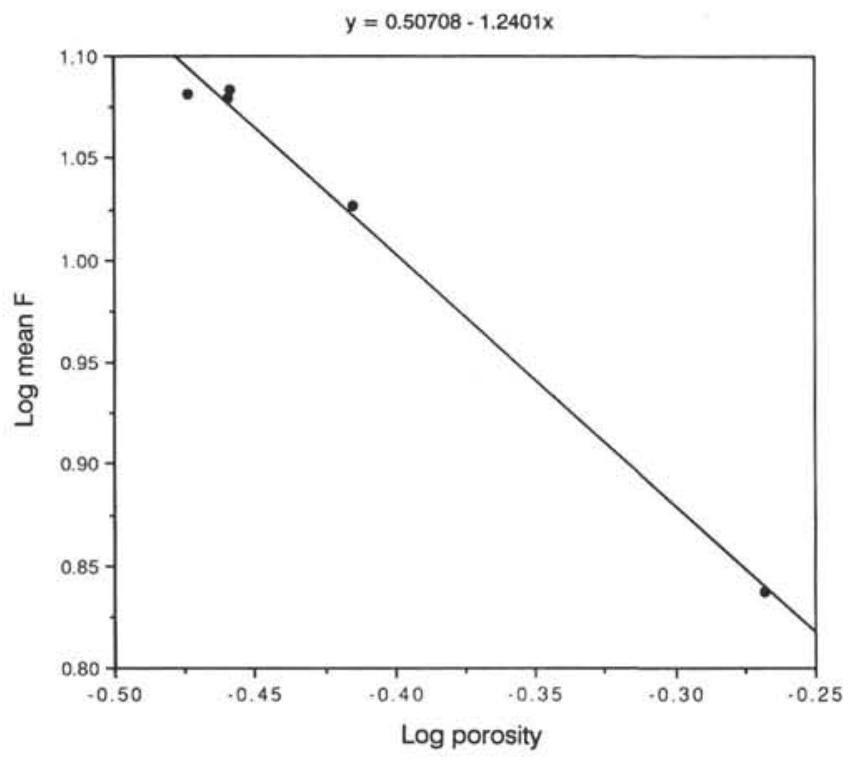

Figure 11. Plot of $\log$ porosity vs. $\log$ mean formation factor for claystones from Units V and VI.

ooze), velocity values begin at about $1.54 \mathrm{~km} / \mathrm{s}$ and increase only to a high of about $1.60 \mathrm{~km} / \mathrm{s}$. Between 360 and $500 \mathrm{mbsf}$, the gradient in the chalks increases sharply to $2.86 \mathrm{~s}^{-1}$. From 600 to 720 mbsf, the gradient becomes negative $\left(-2.5 \mathrm{~s}^{-1}\right)$ as velocity decreases with depth. Between $720-800$ mbsf, the gradient is quite high $\left(11.25 \mathrm{~s}^{-1}\right)$ as velocity increases sharply from 1.9 to 2.8 $\mathrm{km} / \mathrm{s}$. The gradient again becomes negative $\left(-25.0 \mathrm{~s}^{-1}\right)$ below 800 mbsf as the velocity drops in response to the change in lithology from chalk to claystone. An average gradient for the calcareous sequence can be computed using the low velocities at the top of Hole $762 \mathrm{~B}$ and a high velocity at around $800 \mathrm{mbsf}$, near the bottom of the carbonate sequence. The resulting gradient of 1.58 $\mathrm{s}^{-1}$ is close to the average gradient of $1.3 \mathrm{~s}^{-1}$ of Ontong-Java Plateau data of Hamilton (1980).
These changes in velocity with depth are directly proportional to changes in bulk density. Figure 12 is a plot of bulk density vs. mean $V_{p}$ for the lithologic units of Hole $762 \mathrm{C}$. Our range of values agrees with the curve of Hamilton (1980) for calcareous sediments.

\section{NEGATIVE VELOCITY GRADIENT}

Values of $V_{p}$ and $\rho_{b}$ show an average decrease in the chalks between about 600 and 720 mbsf (Fig. 4B). This depth range includes all of Unit IVB and about the first $20 \mathrm{~m}$ of Unit IVC, but this interval does not exhibit significant changes in lithology. Plausible causes for this trend are: (1) gas in the samples (overpressuring), (2) change in mineralogy, or (3) change in microstructure (which would change the porosity). To test the first hypothesis, velocities of 18 samples in the depth range of 594.8-725.8 mbsf were remeasured after samples were resaturated in seawater under a vacuum for $1 \mathrm{hr}$. However, no gas bubbles exited from the samples while they were under vacuum, suggesting that no gas was present. $V_{p}, \rho_{b}$, and $\phi$ values obtained from these samples were identical to the values obtained before resaturation. Therefore, the negative velocity gradient is not caused by overpressuring. Figure 13 shows a plot of mineral content vs. depth for the depth range 600-720 mbsf (data from Wilkens et al., this volume). There appears to be no significant change in mineralogy over this depth range, and shipboard smear slide data suggest no significant change in grain type.

Shipboard porosity data show no significant trend, but shore-based porosity measurements increase slightly over this depth interval (Fig. 5B). Therefore, the negative velocity gradient is most likely due to increasing porosity. Changes in porosity can be caused by either changes in grain size or in microstructure. A decrease in grain size could result in an increase in porosity (Hamilton et al., 1982). Smear slide data indicate that the sediments at this depth are mostly composed of nannofossils and a smaller amount of clay and foraminifera (usually less than 20\%). However, no significant change in grain content occurs over this depth interval. Elevated porosity could also be caused by an open microstructure, which could occur during a period of rapid sedimentation. Sedimentation rates determined during the cruise are not unusually high within this depth range (approximately $1.1 \mathrm{~cm} / \mathrm{k} . \mathrm{y}$. ), but these are only "apparent" sedimentation rates because they are not corrected for compaction.

Although the increase in porosity in this depth range is rather small (from $35 \%$ to $45 \%$ ), the SEM was utilized to determine if a change in microstructure was observable. Two samples were examined: one near the top of the interval at 612.6 mbsf (Sample 122-762C-49X-1, 106-109 $\mathrm{cm}, \phi=$ $35.5 \%$ ) and the other at the bottom at 716.2 mbsf (Sample $122-762 \mathrm{C}-60 \mathrm{X}-1,22-24 \mathrm{~cm}, \phi=45.1 \%$ ). SEM photomicrographs (Plate 1) indicate the grain matrix consists almost exclusively of nannofossils with small amounts of clay. There does not appear to be any obvious microstructural differences between the two samples. But as there is a difference of only $10 \%$ porosity between the two samples, a difference in microstructure may not be apparent. Thus, although SEM observations are inconclusive, an open microstructure would be the more likely reason for the porosity increase over this depth interval.

\section{SUMMARY}

Values of $V_{s}, Q_{p}$, and $\rho^{\prime}$ of 49 samples from Hole $762 \mathrm{C}$ were determined in our acoustics laboratory. These measurements are currently not conducted aboard the JOIDES Resolution. In addition, $V_{p}, \phi, \rho_{b}$, and $\rho_{g}$ were remeasured using alternate methods to those utilized aboard ship. 


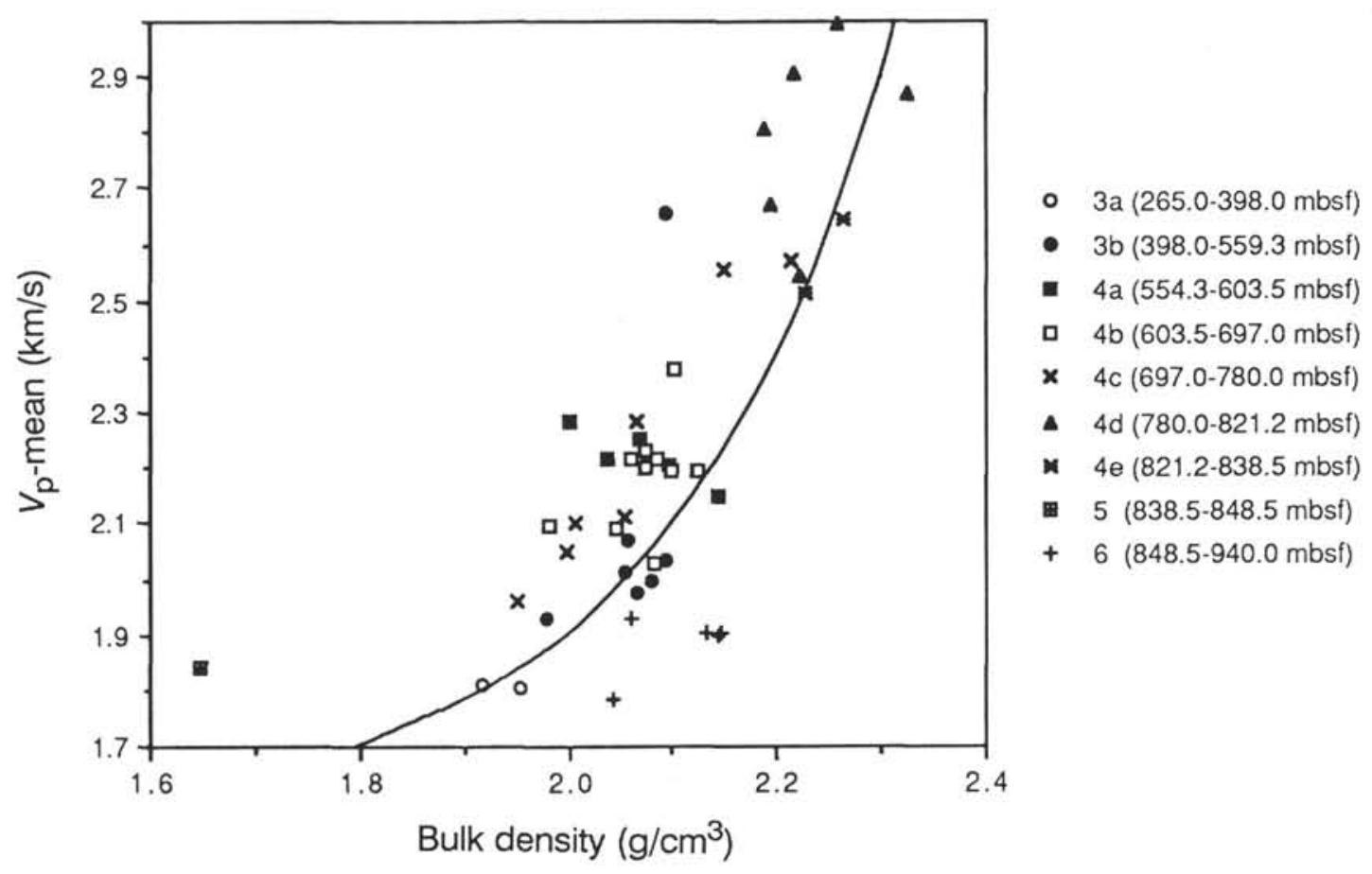

Figure 12. Shore-based bulk density vs. mean $V_{p}$ coded by lithologic unit for Hole $762 \mathrm{C}$. Solid line indicates trend for carbonate sediments from Hamilton (1980).

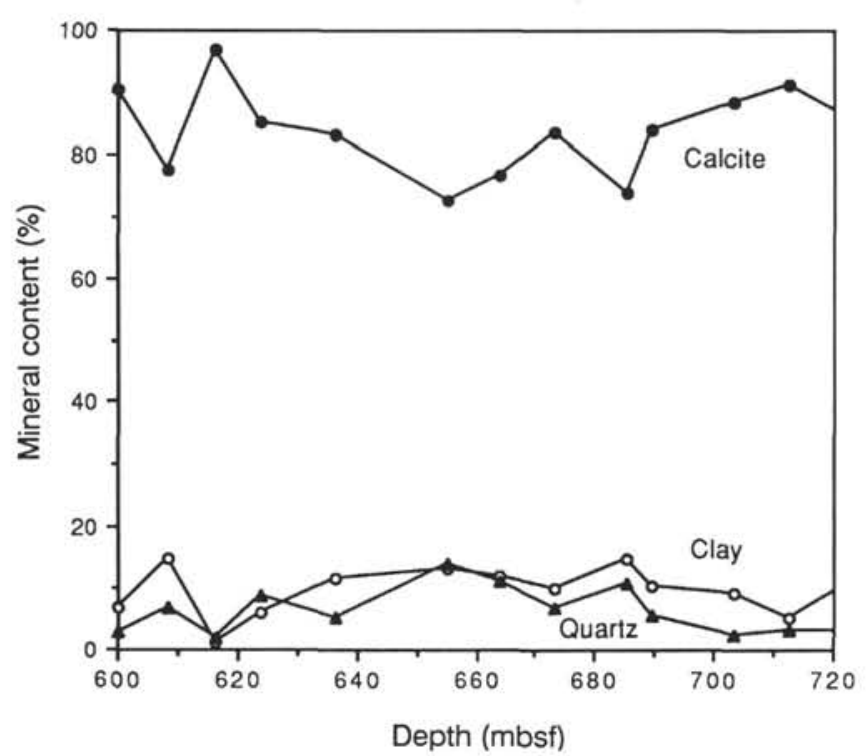

Figure 13. Mineral content of shipboard physical property samples from shore-based X-ray diffraction data in the depth range of $600-720$ mbsf from Hole $762 \mathrm{C}$. Within this depth range velocity decreases with depth, although no significant change in mineralogy is evident.

Shipboard and shore-based $\phi$ and $\rho_{g}$ agree reasonably well, but $V_{p}$ values diverge between 670 and $820 \mathrm{mbsf}$. This was caused by a calibration error in the shipboard Hamilton Frame velocimeter system.

Shipboard $\rho_{b}$ values are consistently higher than shorebased data due to erroneously low volumes determined from wet samples in the shipboard helium-displacement pycnometer. As the pycnometer was not designed to measure wet samples, volumes of wet samples can be determined using the relation: wet volume $=$ dry volume + (wet weight - dry weight). Bulk densities recalculated using wet volumes calculated in this way coincide with shore-based bulk densities. We suggest that ODP use this equation instead of the pycnometer wet volumes.

Cementation factors determined from the Winsaurer equation correctly indicate that the chalk samples are well lithified and the claystone samples are friable. The Winsauer equation apparently does not hold for samples with high porosity.

The negative velocity gradient in the chalks between 600 and 720 mbsf is not caused by overpressuring, because no gas exited from the samples upon restoration in a vacuum. Neither is it caused by change in mineralogy, because mineralogy content is relatively constant within this depth range. It is most likely caused by an increase in porosity that may be due to an open microstructure over this depth interval.

\section{ACKNOWLEDGMENTS}

Juan Lorenzo, Alistair Bent, and Roy Wilkens collected shipboard physical properties data with David O'Brien for Site 762. We are indebted to Roy Wilkens for many helpful discussions about the physical properties of this site, and for providing the sonic log and X-ray diffraction data. We would like to thank Jane Tribble, Suzanne O'Connell, Richard Bachman, and William Busch for critically reviewing this manuscript and for helpful comments. John Balogh maintained the equipment used in the Hawaii physical properties laboratory. Jim Cowen allowed us the use of the SEM, and Don McGee maintained the SEM laboratory. The assistance of the ODP technicians, especially Wendy Autio, Matt Mefferd, Kevin Rogers, and Barry Webber, was greatly appreciated in keeping the shipboard physical properties laboratory operational during Leg 122. This research was supported by JOI/USSAC and the Office of Naval Research. This paper is HIG contribution no. 2392 . 


\section{REFERENCES}

Archie, G. E., 1942. The electrical resistivity log as an aid in determining some reservoir characteristics. Trans. Am. Inst. Min. Metall. Pet. Eng., 146:54-67.

Carlson, R. L., and Christensen, N. I., 1979. Velocity anisotropy in semi-indurated calcareous deep-sea sediments. J. Geophys. Res., $84: 205-211$.

Carlson, R. L., Schaftenaar, C. H., and Moore, R. P., 1983. Causes of compressional-wave anisotropy in calcareous sediments from the Rio Grande Rise. In Barker, P. F., Carlson, R. L., Johnson, D. A., et al., Init. Repts. DSDP, 72: Washington (U.S. Govt. Printing Office), 565-576.

Collett, L. S., and Katsube, T. J., 1973. Electrical parameters of rocks in developing geophysical techniques. Geophysics, 38:79-91.

Exon, N. F., von Rad, U., and von Stackelberg, U., 1982. The geological development of the passive margins of the Exmouth Plateau off northwest Australia. Mar. Geol., 47:131-152.

Ference, M., Lemon, H., and Stephenson, R., 1956. Analytical Experimental Physics: Chicago (Univ. of Chicago Press).

Hamilton, E. L., 1980. Geoacoustic modeling of the seafloor. J. Acoust. Soc. Am., 68:1313-1340.

Hamilton, E. L., Bachman, R. T., Berger, W. H., Johnson, T. C., and Mayer, L. A., 1982. Acoustic and related properties of calcareous deep-sea sediments. J. Sediment. Petrol., 52:733-753.

Haq, B. U., von Rad, U., O'Connell, S., et al., 1990. Proc. ODP, Init. Repts., 122: College Station, TX (Ocean Drilling Program).

Johnson, G. R., 1979. Textural properties. In Hunt, G. R., Johnson, G. R., Olhoeft, G. R., Watson, D. E., and Watson, K. (Eds.), Initial Reports of the Petrophysics Laboratory. Geol. Surv. Circ. (U.S.), 789:67-74.

Johnston, D. H., and Toksoz, M. N., 1980. Ultrasonic $P$ and $S$ wave attenuation in dry and saturated rocks under pressure. J. Geophys. Res., 85:925-936.

Katsube, T. J., 1977. Electrical properties of rocks. Proc. Induced Polarization for Exploration Geologists and Geophysicists, Tempe, AZ (Univ. of Arizona), 15-44.

Kim, D.-C., Katahara, K. W., Manghnani, M. H., and Schlanger, S. O., 1983. Velocity and attenuation in deep-sea carbonate sediments. J. Geophys. Res., 88:2337-2343.
Milholland, P., Manghnani, M. H., Schlanger, S. O., and Sutton, G., 1980. Geoacoustic modeling of deep-sea carbonate sediments. $J$. Acoust. Soc. Am., 68:1351-1360.

O'Brien, D. K., Manghnani, M. H., Tribble, S. J., and Wenk, H.-R., 1989. Preferred orientation and velocity anisotropy in clay-bearing calcareous marine sediments. Eos, 70:1363 (Abstract).

Olhoeft, G. R., 1979. Electrical properties. In Hunt, G. R., Johnson, G. R., Olhoeft, G. R., Watson, D. E., and Watson, K. (Eds.), Initial Reports of the Petrophysics Laboratory. Geol. Surv. Circ. (U.S.), 789:1-26.

Olhoeft, G. R., 1980. Initial report of the petrophysics laboratory, 1977-1979 addendum. Open-File Rep. - U.S. Geol. Surv., 80-522.

Shipboard Scientific Party, 1990. Site 762. In Haq, B. U., von Rad, U., et al., Proc. ODP, Init. Repts., 122: College Station, TX (Ocean Drilling Program), 213-288.

Toksoz, M. N., Johnston, D. H., and Timur, A., 1979. Attenuation of seismic waves in dry and saturated rock: I. Laboratory measurements. Geophysics, 44:681-690.

Ward, S. H., and Fraser, D. C., 1967. Conduction of electricity in rocks. In Hansen, D. A., Heinrichs, W. E., Jr., Holmer, R. C., MacDougall, R. E., Rogers, G. R., Sumner, J. S., and Ward, S. H. (Eds.), Mining Geophysics. Soc. Expl. Geophys., 2:198-223.

Weast, R. C., and Astle, M. J. (Eds.), 1981. CRC Handbook of Chemistry and Physics: Boca Raton, FL (Chemical Rubber Co. Press).

Wilkens, R., McLellan, P., Moran, K., Tribble, J. S., Taylor, E., and Verduzco, E., 1990. Diagenesis and dewatering of clay-rich sediments, Barbados accretionary prism. In Moore, J. C., Mascle, A., et al., Proc. ODP, Sci. Results, 110: College Station, TX (Ocean Drilling Program), 309-320.

Winsauer, W. O., Shearing, H. M., Jr., Masson, P. H., and Williams, M., 1952. Resistivity of brine saturated sands in relation to pore geometry. AAPG Bull., 36:253-277.

Zamanek, J., Jr., and Rudnick, I., 1961. Attenuation of elastic waves. J. Acoust. Soc. Am., 33:1283-1288.

Date of initial receipt: 2 May 1990

Date of acceptance: 16 January 1991

Ms 122B-115 
A

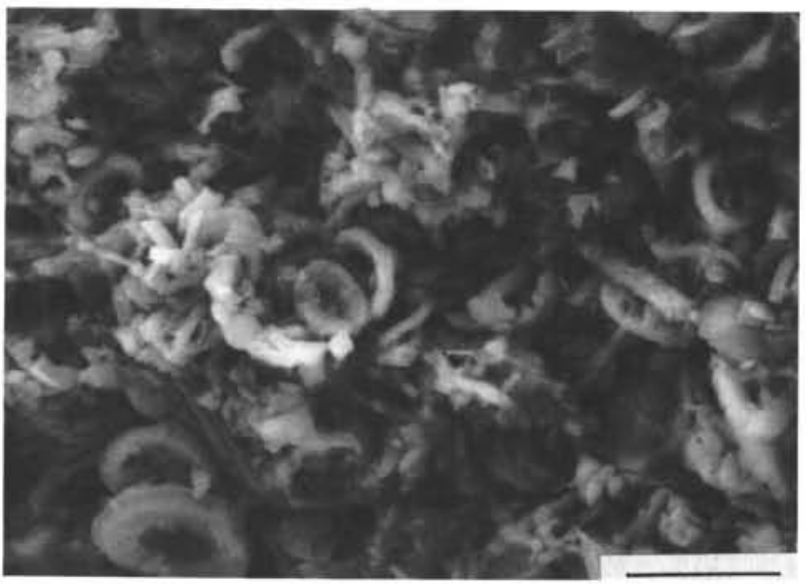

C

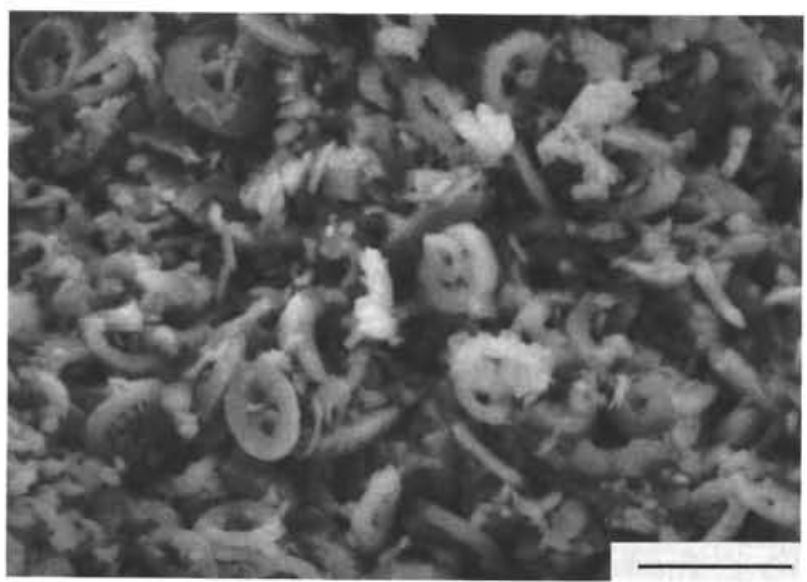

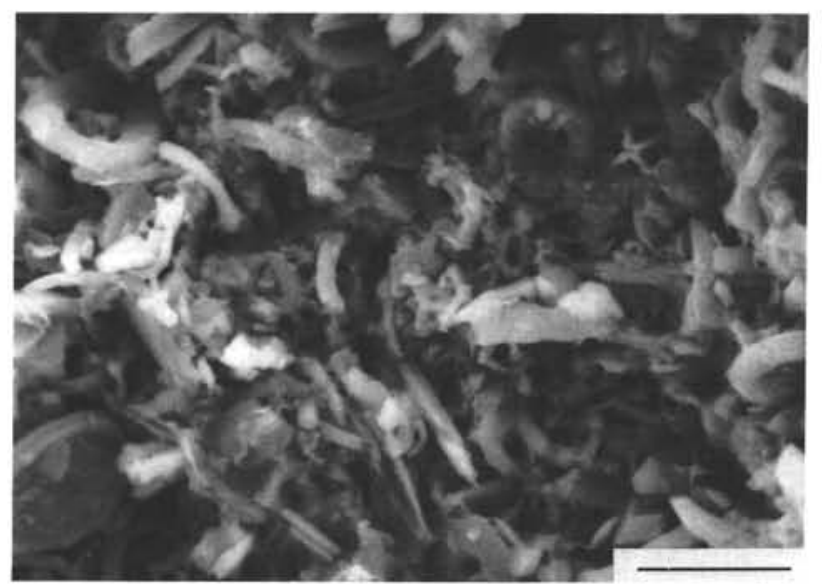

B
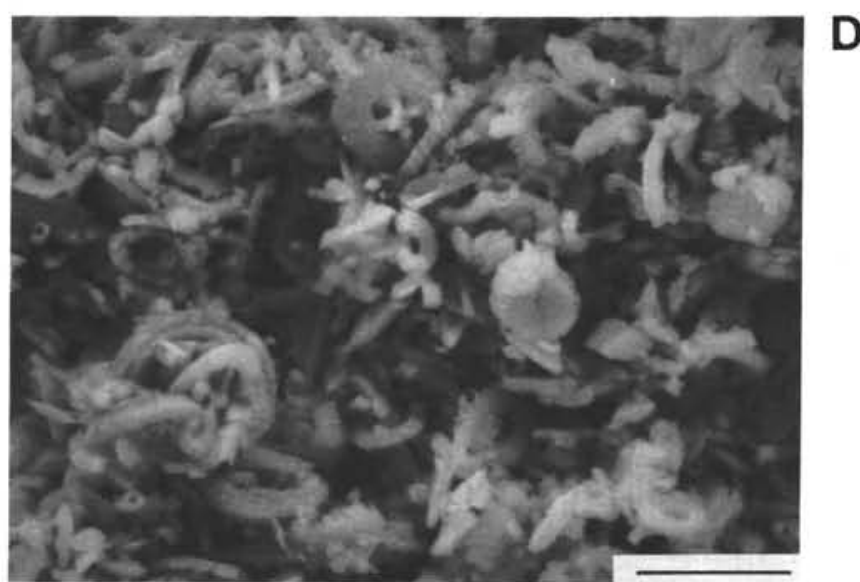

Plate 1. SEM micrographs. A, B. Sample 122-762C-49X-1, 106-109 cm. C, D. Sample 122-762C-60X-1, 22-24 cm. Sample 122-762-49X-1, $106-109 \mathrm{~cm}$, is at the top of the negative velocity gradient and Sample 122-762-60X-1, 22-24 cm, is at the bottom. There does not appear to be any obvious difference in microstructure between the two samples. Scale bars $=10 \mu \mathrm{m}$. 